\title{
Generalized derivatives for the solution operator of the obstacle problem
}

\author{
Anne-Therese Rauls* Gerd Wachsmuth ${ }^{\dagger}$ iD
}

June 14, 2018

\begin{abstract}
We characterize generalized derivatives of the solution operator of the obstacle problem. This precise characterization requires the usage of the theory of so-called capacitary measures and the associated solution operators of relaxed Dirichlet problems. The generalized derivatives can be used to obtain a novel necessary optimality condition for the optimal control of the obstacle problem with control constraints. A comparison shows that this system is stronger than the known system of C-stationarity.
\end{abstract}

Keywords: obstacle problem, generalized derivative, capacitary measure, relaxed Dirichlet problem, C-stationarity

MSC: 49K40, 47J20, 49J52, 58C20

\section{Introduction}

We consider the obstacle problem

$$
\text { Find } y \in K: \quad\langle-\Delta y-u, z-y\rangle \geq 0 \quad \forall z \in K .
$$

Here, $\Omega \subset \mathbb{R}^{d}$ is a bounded, open set and the closed, convex set $K \subset H_{0}^{1}(\Omega)$ is given by

$$
K:=\left\{v \in H_{0}^{1}(\Omega) \mid v \geq \psi \text { q.e. on } \Omega\right\}
$$

where $\psi: \Omega \rightarrow[-\infty, \infty)$ is a given quasi upper-semicontinuous function. We assume $K \neq \emptyset$. It is well known that for each $u \in H^{-1}(\Omega)$ there exists a unique solution

\footnotetext{
${ }^{*}$ Technische Universität Darmstadt, Department of Mathematics, Research Group Optimization, 64293 Darmstadt, Germany, http://www3.mathematik.tu-darmstadt.de/hp/ag-optimierung/ rauls-anne-therese/startseite.html, rauls@mathematik.tu-darmstadt.de

${ }^{\dagger}$ Brandenburgische Technische Universität Cottbus-Senftenberg, Institute of Mathematics, Chair of Optimal Control, 03046 Cottbus, Germany, https://www.b-tu.de/fg-optimale-steuerung, gerd. wachsmuth@b-tu.de
} 
$y:=S(u) \in H_{0}^{1}(\Omega)$ of $(1)$. Moreover, the mapping $S: H^{-1}(\Omega) \rightarrow H_{0}^{1}(\Omega)$ is globally Lipschitz continuous.

Our main goal is the characterization of so-called generalized derivatives of the mapping $S$. That is, given $u \in H^{-1}(\Omega)$, we are going to characterize the limit points of $S^{\prime}\left(u_{n}\right)$, where $\left\{u_{n}\right\}$ is a sequence of points in which $S$ is Gâteaux differentiable and which converge towards $u$. Since the involved spaces are infinite dimensional, there is some choice concerning the topologies. We will equip the space of operators with the weak or the strong operator topology and on $H^{-1}(\Omega)$ we use the weak or strong topology. When considering the weak topology on $H^{-1}(\Omega)$, we also require that $\left\{S\left(u_{n}\right)\right\}$ converges weakly to $S(u)$. At this point we also recall the famous result [Mignot, 1976, Théorème 1.2] which shows that $S$ is Gâteaux differentiable on a dense subset of $H^{-1}(\Omega)$. Thus, each point $u \in H^{-1}(\Omega)$ can be approximated by differentiability points of $S$.

The precise characterization of these generalized derivatives will involve the notion of "capacitary measures" and "relaxed Dirichlet problems". A comprehensive introduction to these topics will be given in Section 3 below. A Borel measure is a $\sigma$-additive set function on the Borel $\sigma$-algebra with values in $[0, \infty]$. A capacitary measure $\mu$ is a Borel measure which does not charge sets of capacity zero and which satisfies a regularity condition, see Definition 3.1. For each capacitary measure $\mu$, we can consider the solution operator $u \mapsto y$ of

$$
-\Delta y+\mu y=u
$$

equipped with homogeneous Dirichlet boundary conditions, see Section 3 for the precise definition of this solution operator. It is well known that the solution operator of (2) can be approximated (in the weak operator topology) by the solution operators of

$$
-\Delta y=u \text { in } H^{-1}\left(\Omega_{n}\right), \quad y \in H_{0}^{1}\left(\Omega_{n}\right)
$$

for some sequence of open sets $\Omega_{n} \subset \Omega$. Moreover, each sequence of solution operators of (3) converges (along a subsequence) to a solution operator of (3) with an appropriate capacitary measure $\mu$. This motivates to term (2) a relaxed Dirichlet problem. Our analysis reveals that the generalized derivatives of $S$ are precisely sets of solution operators of (2) with appropriate conditions on $\mu$.

After we have established the characterization of the generalized derivatives, we turn our attention to the optimal control of the obstacle problem

$$
\text { Minimize } \quad J(y, u) \quad \text { with } y=S(u) \text { and } u \in U_{\mathrm{ad}} .
$$

Here, $J: H_{0}^{1}(\Omega) \times L^{2}(\Omega) \rightarrow \mathbb{R}$ is assumed to be Fréchet differentiable with partial derivatives $J_{y}$ and $J_{u}$, and $U_{\text {ad }} \subset L^{2}(\Omega)$ is assumed to be closed and convex. By a formal application of Lagrange duality, we arrive at the stationarity system

$$
0 \in L^{\star} J_{y}(y, u)+J_{u}(y, u)+\mathcal{N}_{U_{\mathrm{ad}}}(u) \quad \text { for some } L \in \partial_{B} S(u) .
$$

Here, $\partial_{B} S(u)$ is a generalized differential of $S$ at $u$, and $\mathcal{N}_{U_{\text {ad }}(u)}$ is the normal cone in the sense of convex analysis of $U_{\text {ad }}$ at $u$. We will see that (for a certain choice of the involved topologies in the definition of $\left.\partial_{B} S(u)\right)$ this system is slightly stronger than the so-called 
system of C-stationarity from [Schiela, D. Wachsmuth, 2013]. Moreover, by inspecting the proof of [Schiela, D. Wachsmuth, 2013], it is possible to strengthen this system of C-stationarity such that it becomes equivalent to (5). Therefore, our research leads to the discovery of a new necessary optimality condition for (4) which improves the known system of C-stationarity.

We put our work into perspective. Our research was highly influenced by the recent contribution [Christof et al., 2018]. Therein, the authors considered the non-smooth partial differential equation

$$
-\Delta y+\max \{y, 0\}=u
$$

equipped with homogeneous Dirichlet boundary conditions. They characterized generalized derivatives for the solution operator mapping $u \mapsto y$. Subsequently, these generalized derivatives are used to derive and compare optimality conditions for the optimal control of (6). Furthermore, a single generalized gradient for the infinite-dimensional obstacle problem was computed in [Rauls, Ulbrich, 2018]. This gradient is contained in all of the generalized derivatives that we will consider and the approach gives a hint how the generalized differential involving strong topologies might look like. The derivation there uses different tools and while being able to treat also the variational inequality

$$
\text { Find } y \in K: \quad\langle-\Delta y-f(u), z-y\rangle \geq 0 \quad \forall z \in K .
$$

for an appropriate monotone operator $f$ with range smaller than $H^{-1}(\Omega)$, it is hard to characterize the entire generalized differential involving strong topologies with this approach, let alone those involving also weak topologies. We are not aware of any other contribution in which generalized derivatives of nonsmooth infinite-dimensional mappings are computed. There is, however, a vast amount of literature in the finite-dimensional setting. We only mention [Klatte, Kummer, 2002; Outrata et al., 1998].

Let us give an outline of this work. In the following section, we recall the relevant notions and results from capacity theory (Section 2.1), recapitulate differentiability properties of the obstacle problem (Section 2.2) and introduce the generalized differentials we are dealing with in this paper (Section 2.3). We review the concepts of capacitary measures, relaxed Dirichlet problems and $\gamma$-convergence in Section 3. The generalized differentials of the solution operator to the obstacle problem associated to the strong operator topology will be established in Section 4. Under additional regularity assumptions we characterize the generalized differential involving the strong topology in $H^{-1}(\Omega)$ and the weak operator topology for the operators in Section 5. In Section 6, we give an example to show that the generalized differential involving only weak topologies can be very large, even in points of differentiability. Based on the developed characterizations of generalized derivatives, we discuss stationarity systems for the optimal control of the obstacle problem with control constraints in Section 7.

\section{Notation and known results}

In this work, $\Omega \subset \mathbb{R}^{d}$ is an open bounded set in dimension $d \geq 2$. By $H_{0}^{1}(\Omega)$, we denote the usual Sobolev space. Its norm is given by $\|u\|_{H_{0}^{1}(\Omega)}^{2}=\int_{\Omega}|\nabla u|^{2} \mathrm{~d} x$ and the duality 
pairing between $H^{-1}(\Omega):=H_{0}^{1}(\Omega)^{\star}$ and $H_{0}^{1}(\Omega)$ is $\langle\cdot, \cdot\rangle$.

We often deal with subsets of $\Omega$ that are defined only up to a set of capacity zero, see also Section 2.1. As a consequence, relations between such sets, such as inclusions and equalities, are meaningful only up to a set of capacity zero. For subsets $B, C$ that are defined up to capacity zero, we distinguish such relations by writing $B \subset_{q} C, B \supset_{q} C$ or $B={ }_{q} C$. Similarly, definitions of sets up to capacity zero, such as the zero set of a family of quasi-continuous representatives, see Section 2.1, are denoted by " $:=q$ ".

\subsection{Introduction to capacity theory}

We collect some fundamentals on capacity theory. For the definitions, see e.g. [Attouch et al., 2014, Sections 5.8.2, 5.8.3], [Delfour, Zolésio, 2011, Definition 6.2] or [Bonnans, Shapiro, 2000, Definition 6.47].

Definition 2.1. (i) For every set $A \subset \Omega$ the capacity (in the sense of $H_{0}^{1}(\Omega)$ ) is defined as

$$
\operatorname{cap}(A):=\inf \left\{\|u\|_{H_{0}^{1}(\Omega)}^{2}: u \in H_{0}^{1}(\Omega), u \geq 1 \text { a.e. in a neighborhood of } A\right\} .
$$

(ii) A subset $\hat{\Omega} \subset \Omega$ is called quasi-open if for all $\varepsilon>0$ there is an open set $O_{\varepsilon} \subset \Omega$ with $\operatorname{cap}\left(O_{\varepsilon}\right)<\varepsilon$ such that $\hat{\Omega} \cup O_{\varepsilon}$ is open. The relative complement of a quasi-open set in $\Omega$ is called quasi-closed.

(iii) A function $v: \Omega \rightarrow \overline{\mathbb{R}}=[-\infty,+\infty]$ is called quasi-continuous (quasi lower-semicontinuous, quasi upper-semicontinuous, respectively) if for all $\varepsilon>0$ there is an open set $O_{\varepsilon} \subset \Omega$ with $\operatorname{cap}\left(O_{\varepsilon}\right)<\varepsilon$ such that $v$ is continuous (lower-semicontinuous, upper-semicontinuous, respectively) on $\Omega \backslash O_{\varepsilon}$.

If a property holds on $\Omega$ except on a set of zero capacity, we say that this property holds quasi-everywhere (q.e.) in $\Omega$. It is well known that each $v \in H_{0}^{1}(\Omega)$ possesses a quasi-continuous representative, which is uniquely determined up to values on a set of zero capacity, see e.g. [Bonnans, Shapiro, 2000, Lemma 6.50] or [Delfour, Zolésio, 2011, Chapter 8, Theorem 6.1]. Moreover, the proof in the former reference yields that this representative can be chosen to be even Borel measurable. From now on, we will always use quasi-continuous and Borel measurable representatives when working with functions from $H_{0}^{1}(\Omega)$.

Similarly, every quasi lower-/upper-semicontinuous function can be made Borel measurable by a modification on a set of capacity zero. Indeed, for a quasi upper-semicontinuous function $\psi$, the sets $\{\psi<q\}$ are quasi-open for all $q \in \mathbb{Q}$. Hence, there are Borel sets $O_{q}$ of capacity zero, such that $\{\psi<q\} \cup O_{q}$ is a Borel set for each $q \in \mathbb{Q}$. By setting $\psi$ to $-\infty$ on $\bigcup_{q \in \mathbb{Q}} O_{q}$, the function is still quasi upper-semicontinuous and becomes Borel measurable. W.l.o.g., we will assume that the obstacle $\psi$ is Borel measurable.

Lemma 2.2. Let $\left\{v_{n}\right\} \subset H_{0}^{1}(\Omega), v \in H_{0}^{1}(\Omega)$ and assume that $v_{n} \rightarrow v$ in $H_{0}^{1}(\Omega)$. Then there is a subsequence of $\left\{v_{n}\right\}$, such that the sequence (of quasi-continuous representatives of) $\left\{v_{n}\right\}$ converges pointwise quasi-everywhere to (the quasi-continuous representative of) $v$. 
Proof. See [Bonnans, Shapiro, 2000, Lemma 6.52].

It is possible to extend the Sobolev space $H_{0}^{1}(\Omega)$ to quasi-open subsets $\hat{\Omega} \subset \Omega$ by setting

$$
H_{0}^{1}(\hat{\Omega}):=\left\{v \in H_{0}^{1}(\Omega): v=0 \text { q.e. on } \Omega \backslash \hat{\Omega}\right\} .
$$

We point out that this definition is consistent with the usual definition of $H_{0}^{1}(\hat{\Omega})$ in the case that $\hat{\Omega}$ is open, see [Heinonen et al., 1993, Theorem 4.5]. By Lemma 2.2, the space $H_{0}^{1}(\hat{\Omega})$ is a closed subspace of $H_{0}^{1}(\Omega)$.

Lemma 2.3. Let $\hat{\Omega} \subset \Omega$ be quasi-open and assume there is a sequence of quasi-open sets $\left\{\hat{\Omega}_{n}\right\}$ such that $\left\{\hat{\Omega}_{n}\right\}$ is increasing in $n$ and such that $\hat{\Omega}={ }_{q} \bigcup_{n=1}^{\infty} \hat{\Omega}_{n}$. Let $v \in H_{0}^{1}(\hat{\Omega})$. Then there is a sequence $\left\{v_{n}\right\}$ with $v_{n} \in H_{0}^{1}\left(\hat{\Omega}_{n}\right)$ for each $n \in \mathbb{N}$ such that $v_{n} \rightarrow v$ in $H_{0}^{1}(\Omega)$. Furthermore, it holds $\sup \left|v_{n}\right| \leq \sup |v|$.

Proof. The sequence $\left\{\hat{\Omega}_{n}\right\}$ represents a quasi-covering of $\hat{\Omega}$, therefore, combining [Kilpeläinen, Malý, 1992, Theorem 2.10 and Lemma 2.4], we find a sequence $\left\{v_{n}\right\}$ such that $v_{n} \rightarrow v$ in $H_{0}^{1}(\Omega)$ and such that each $v_{n}$ is a finite sum of elements in $\bigcup_{m=1}^{\infty} H_{0}^{1}\left(\hat{\Omega}_{m}\right)$. Furthermore, $\sup \left|v_{n}\right| \leq \sup |v|$. Since the sets $\hat{\Omega}_{n}$ are increasing, for each $n \in \mathbb{N}$ there is $j \in \mathbb{N}$ such that $v_{n} \in \bigcap_{m=j}^{\infty} H_{0}^{1}\left(\hat{\Omega}_{m}\right)$. We extend the sequence by adding copies of elements in $\left\{v_{n}\right\}$ to the original sequence. This yields a sequence with the desired properties.

Using the same ideas, we can characterize the sum of two Sobolev spaces on quasi-open domains.

Lemma 2.4. Let $\Omega_{1}, \Omega_{2} \subset \Omega$ be quasi-open. Then,

$$
{\overline{H_{0}^{1}}\left(\Omega_{1}\right)+H_{0}^{1}\left(\Omega_{2}\right)}^{H_{0}^{1}(\Omega)}=H_{0}^{1}\left(\Omega_{1} \cup \Omega_{2}\right) .
$$

Moreover, for every $v \in H_{0}^{1}\left(\Omega_{1} \cup \Omega_{2}\right)^{+}$, there exist sequences $\left\{v_{n}^{(1)}\right\} \subset H_{0}^{1}\left(\Omega_{1}\right)^{+}$and $\left\{v_{n}^{(2)}\right\} \subset H_{0}^{1}\left(\Omega_{2}\right)^{+}$with $0 \leq v_{n}^{(1)}+v_{n}^{(2)} \leq v$ q.e. on $\Omega$ for all $n \in \mathbb{N}$, and $v_{n}^{(1)}+v_{n}^{(2)} \rightarrow v$ in $H_{0}^{1}(\Omega)$.

Proof. Since $\left\{\Omega_{1}, \Omega_{2}\right\}$ is a quasi-covering of $\Omega_{1} \cup \Omega_{2}$, we can argue as in the proof of Lemma 2.3 to obtain the first identity. For the second assertion, an inspection of the proofs of [Kilpeläinen, Malý, 1992, Theorem 2.10 and Lemma 2.4] shows that the approximating functions can be chosen to be pointwise bounded by 0 and $v$.

We also recall that positive elements in the dual space $H^{-1}(\Omega)$ of $H_{0}^{1}(\Omega)$ can be identified with regular Borel measures which are finite on compact sets. Here, a Borel measure on $\Omega$ is a measure over the Borel $\sigma$-algebra $\mathcal{B}$, which is the smallest $\sigma$-algebra containing all open subsets of $\Omega$. We call a Borel measure $\mu$ regular if

$$
\mu(B)=\inf \{\mu(O): B \subset O, O \text { is open }\}=\sup \{\mu(C): C \subset B, C \text { is compact }\}
$$

holds for all $B \in \mathcal{B}$. Finally, $\mu$ is said to be finite on compact sets, if $\mu(K)<+\infty$ for all compact subsets $K \subset \Omega$. 
Lemma 2.5. Let $\xi \in H^{-1}(\Omega)^{+}$be given, i.e., $\langle\xi, v\rangle \geq 0$ for all $v \in H_{0}^{1}(\Omega)$ with $v \geq 0$.

(i) The functional $\xi$ can be identified with a regular Borel measure on $\Omega$ which is finite on compact sets and which possesses the following property: For every Borel set $B \subset \Omega$ with $\operatorname{cap}(B)=0$, we have $\xi(B)=0$.

(ii) Every function $v \in H_{0}^{1}(\Omega)$ is $\xi$-integrable and it holds

$$
\langle\xi, v\rangle_{H^{-1}(\Omega), H_{0}^{1}(\Omega)}=\int_{\Omega} v \mathrm{~d} \xi .
$$

(iii) There is a quasi-closed set $\mathrm{f}-\operatorname{supp}(\xi) \subset \Omega$ with the property that for all $v \in H_{0}^{1}(\Omega)^{+}$ it holds $\langle\xi, v\rangle_{H^{-1}(\Omega), H_{0}^{1}(\Omega)}=0$ if and only if $v=0$ q.e. on $\mathrm{f}-\operatorname{supp}(\xi)$. The set $\mathrm{f}-\operatorname{supp}(\xi)$ is uniquely defined up to a set of zero capacity.

Proofs for statement (i) and (ii) can be found in [Bonnans, Shapiro, 2000, p. 564, 565]. Note that the regularity of $\mu$ is implied by the property of being finite on compact sets, see [Rudin, 1987, Theorem 2.18]. For part (iii) we refer to [Harder, G. Wachsmuth, 2018, Lemma 3.7]. See also [Harder, G. Wachsmuth, 2018, Lemma 3.5] and for a different description of the fine support $f-\operatorname{supp}(\xi)$ in (iii) see [G. Wachsmuth, 2014, Lemma A.4].

\subsection{Differentiability of the solution operator of the obstacle problem}

For the variational inequality (1), we consider the solution operator $S: H^{-1}(\Omega) \rightarrow H_{0}^{1}(\Omega)$ that maps $u \in H^{-1}(\Omega)$ to the unique solution $y=S(u)$ of $(1)$. We define the active set associated with $u \in H^{-1}(\Omega)$ by

$$
A(u):={ }_{q}\{\omega \in \Omega: S(u)(\omega)=\psi(\omega)\}
$$

and the inactive set by

$$
I(u):={ }_{q} \Omega \backslash A(u) .
$$

We emphasize that these sets are defined up to sets of capacity zero since we always work with the quasi-continuous representatives of functions from $H_{0}^{1}(\Omega)$, see also Section 2.1 above. Furthermore, $A(u)$ is quasi-closed, $I(u)$ is quasi-open and both sets are Borel measurable.

It is well known that $S$ is directionally differentiable and that the directional derivative at $u \in H^{-1}(\Omega)$ in direction $h \in H^{-1}(\Omega)$, which is denoted by $S^{\prime}(u ; h)$, solves the variational inequality

$$
\text { Find } y \in \mathcal{K}(u): \quad\langle-\Delta y-h, z-y\rangle \geq 0 \quad \forall z \in \mathcal{K}(u),
$$

see [Mignot, 1976]. Here, $\mathcal{K}(u)$ denotes the critical cone, which, according to [G. Wachsmuth, 2014, Lemma 3.1], has the following structure:

$$
\mathcal{K}(u):=\left\{z \in H_{0}^{1}(\Omega): z \geq 0 \text { q.e. on } A(u) \text { and } z=0 \text { q.e. on } A_{s}(u)\right\} .
$$


Here, the strictly active set $A_{s}(u)$ is a quasi-closed subset of the active set $A(u)$. It has a representation in terms of the fine support of the multiplier $\xi=-\Delta S(u)-u \in H^{-1}(\Omega)^{+}$, see [G. Wachsmuth, 2014, Appendix A]. In fact, it holds

$$
A_{s}(u)={ }_{q} \mathrm{f}-\operatorname{supp}(\xi) .
$$

Again, we emphasize that this definition is unique up to a subset of capacity zero.

The following lemma characterizes the points in which $S$ is Gâteaux differentiable.

Lemma 2.6. The solution operator $S$ of the obstacle problem (1) is Gâteaux differentiable in $u \in H^{-1}(\Omega)$ if and only if the strict complementarity condition is valid in u, i.e., if and only if the equality $A(u)={ }_{q} A_{s}(u)$ holds.

Proof. Assume that $A(u)={ }_{q} A_{s}(u)$ holds. Then $\mathcal{K}(u)=\left\{z \in H_{0}^{1}(\Omega): z=0\right.$ q.e. on $\left.A(u)\right\}$ is a linear subspace and the variational inequality $(7)$ for the directional derivative $S^{\prime}(u ; h)$ reduces to

$$
\text { Find } y \in \mathcal{K}(u): \quad\langle-\Delta y-h, z\rangle=0 \quad \forall z \in \mathcal{K}(u),
$$

i.e., $S^{\prime}(u ; \cdot)$ is linear and bounded.

For the reverse implication, assume that $S$ is Gâteaux differentiable in $u \in H^{-1}(\Omega)$. By the variational inequality (7) we obtain that the image of $S^{\prime}(u ; \cdot)$ is contained in $\mathcal{K}(u)$. Conversely, let $v \in \mathcal{K}(u)$ be arbitrary. Then we can check $v=S^{\prime}(u ;-\Delta v)$, which implies that $\mathcal{K}(u)$ coincides with the image of $S^{\prime}(u ; \cdot)$. Thus, $\mathcal{K}(u)$ is a linear subspace of $H_{0}^{1}(\Omega)$. Using $A_{s}(u) \subset_{q} A(u)$, we trivially have

$$
H_{0}^{1}(\Omega \backslash A(u)) \subset \mathcal{K}(u) \subset H_{0}^{1}\left(\Omega \backslash A_{s}(u)\right) .
$$

Now, for $v \in H_{0}^{1}\left(\Omega \backslash A_{s}(u)\right)^{+}$, we have $v \geq 0$ q.e. in $\Omega$. Hence, $v \in \mathcal{K}(u)$ and, since $\mathcal{K}(u)$ is a subspace, we also have $-v \in \mathcal{K}(u)$. This leads to $v \geq 0$ and $v \leq 0$ q.e. on $A(u)$. Therefore, $v \in H_{0}^{1}(\Omega \backslash A(u))$. This shows

$$
H_{0}^{1}(\Omega \backslash A(u))=\mathcal{K}(u)=H_{0}^{1}\left(\Omega \backslash A_{s}(u)\right) .
$$

Finally, Theorem 3.9 below implies that the equality $A(u)={ }_{q} A_{s}(u)$ holds.

To summarize, the Gâteaux derivative of the solution operator of the obstacle problem in differentiability points $u \in H^{-1}(\Omega)$ is given by the operator $L_{I(u)} \in \mathcal{L}\left(H^{-1}(\Omega), H_{0}^{1}(\Omega)\right)$, where for $h \in H^{-1}(\Omega)$, the element $L_{I(u)}(h)$ is the solution to the boundary value problem

$$
y \in H_{0}^{1}(I(u)): \quad-\Delta y=h .
$$

This equality has to be understood in the sense of $H_{0}^{1}(I(u))^{\star}$, i.e., $\langle-\Delta y-h, v\rangle=0$ for all $v \in H_{0}^{1}(I(u))$. 


\subsection{Generalized differentials}

The generalized differentials, which we will consider, consist of operators in $\mathcal{L}(X, Y)$. In their definition, we will differentiate between different topologies on $X$ and $\mathcal{L}(X, Y)$. We consider the following standard operator topologies on $\mathcal{L}(X, Y)$.

Definition 2.7. Let $X$ and $Y$ be Banach spaces and $\left\{L_{n}\right\}, L \subset \mathcal{L}(X, Y)$.

(i) We say that the sequence $\left\{L_{n}\right\}$ converges to $L$ in the strong operator topology (SOT) if and only if $\left\{L_{n} h\right\}$ converges to Lh in $Y$ for all $h \in X$. If $\left\{L_{n}\right\}$ converges to $L$ in the strong operator topology, we write $L_{n} \stackrel{\text { SOT }}{\longrightarrow} L$.

(ii) We say that the sequence $\left\{L_{n}\right\}$ converges to $L$ in the weak operator topology (WOT) if and only if $\left\{L_{n} h\right\}$ converges to Lh weakly in $Y$ for all $h \in X$. If $\left\{L_{n}\right\}$ converges to $L$ in the weak operator topology, we write $L_{n} \stackrel{\text { WOT }}{\longrightarrow} L$.

From the uniform boundedness principle, we obtain that a sequence of operators which converges in WOT has to be bounded.

Lemma 2.8. Let $\left\{L_{n}\right\} \subset \mathcal{L}(X, Y)$ and assume that $L_{n} \stackrel{\text { wOT }}{\longrightarrow} L$ for some $L \in \mathcal{L}(X, Y)$. Then there is a constant $C>0$ such that $\left\|L_{n}\right\|_{\mathcal{L}(X, Y)} \leq C$ for all $n \in \mathbb{N}$.

The next lemma shows under which conditions a product $L_{n} h_{n}$ converges.

Lemma 2.9. Let $\left\{L_{n}\right\} \subset \mathcal{L}(X, Y)$ and $\left\{h_{n}\right\} \subset X$ be sequences.

(i) If $L_{n} \stackrel{\mathrm{SOT}}{\longrightarrow} L$ and $h_{n} \rightarrow h$ then $L_{n} h_{n} \rightarrow L h$.

(ii) If $L_{n} \stackrel{\text { WOT }}{\longrightarrow} L$ and $h_{n} \rightarrow h$ then $L_{n} h_{n} \rightarrow L h$.

(iii) If $L_{n} \stackrel{\mathrm{WOT}}{\longrightarrow} L, L_{n}^{\star} \stackrel{\mathrm{SOT}}{\longrightarrow} L^{\star}$ and $h_{n} \rightarrow h$ then $L_{n} h_{n} \rightarrow L h$.

Proof. In any case, the norm of the operators $L_{n}$ is uniformly bounded, see Lemma 2.8 . Now, we use the identity

$$
L_{n} h_{n}-L h=\left(L_{n} h-L h\right)+L_{n}\left(h_{n}-h\right) .
$$

In cases (i) and (ii), the claim follows immediately.

In case (iii), $L_{n} h-L h \rightarrow 0$ is clear. To prove the weak convergence of the second addend, we take $f \in Y^{\star}$ and have

$$
\left\langle f, L_{n}\left(h_{n}-h\right)\right\rangle_{Y^{\star}, Y}=\left\langle L_{n}^{\star} f, h_{n}-h\right\rangle_{X^{\star}, X} \rightarrow 0
$$

since $L_{n}^{\star} \stackrel{\mathrm{SOT}}{\longrightarrow} L^{\star}$ by assumption.

Now we define the generalized derivatives that we will deal with in this paper. 
Definition 2.10. Let $T: X \rightarrow Y$ be a locally Lipschitz mapping from a separable Banach space $X$ to a separable and reflexive Banach space $Y$. We denote the set of points in $X$ in which $T$ is Gâteaux differentiable by $D_{T}$. For $x \in X$ we define the following generalized derivatives

$$
\begin{array}{r}
\partial_{B}^{s s} T(x):=\left\{L \in \mathcal{L}(X, Y): \exists\left\{x_{n}\right\} \subset D_{T}: x_{n} \rightarrow x \text { in } X, T^{\prime}\left(x_{n}\right) \stackrel{\text { SOT }}{\longrightarrow} L \text { in } \mathcal{L}(X, Y)\right\}, \\
\partial_{B}^{s w} T(x):=\left\{L \in \mathcal{L}(X, Y): \exists\left\{x_{n}\right\} \subset D_{T}: x_{n} \rightarrow x \text { in } X, T^{\prime}\left(x_{n}\right) \stackrel{\text { WOT }}{\longrightarrow} L \text { in } \mathcal{L}(X, Y)\right\}, \\
\partial_{B}^{w s} T(x):=\left\{L \in \mathcal{L}(X, Y): \exists\left\{x_{n}\right\} \subset D_{T}: x_{n} \rightarrow x \text { in } X, T\left(x_{n}\right) \rightarrow T(x) \text { in } Y,\right. \\
\left.T^{\prime}\left(x_{n}\right) \stackrel{\text { SOT }}{\longrightarrow} L \text { in } \mathcal{L}(X, Y)\right\}, \\
\partial_{B}^{w w} T(x):=\left\{L \in \mathcal{L}(X, Y): \exists\left\{x_{n}\right\} \subset D_{T}: x_{n} \rightarrow x \text { in } X, T\left(x_{n}\right) \rightarrow T(x) \text { in } Y,\right. \\
\left.T^{\prime}\left(x_{n}\right) \stackrel{\text { WOT }}{\longrightarrow} L \text { in } \mathcal{L}(X, Y)\right\} .
\end{array}
$$

Note that the first superscript refers to the mode of convergence of the points $x_{n}$ in $X$, whereas the second superscript refers to the type of operator topology for the convergence of $T^{\prime}\left(x_{n}\right)$.

In the literature, these generalized differentials are sometimes called "subderivatives". However, this notion is only senseful for functions mapping into $\mathbb{R}$ (or, more generally, into an ordered set).

Note that, in contrast to [Christof et al., 2018, Definition 3.1], we also require that the values $\left\{T\left(x_{n}\right)\right\}$ converge weakly to $T(x)$ when considering the generalized differentials $\partial_{B}^{w s} T(x)$ and $\partial_{B}^{w w} T(x)$. Since the solution operator $\tilde{S}$ to the non-smooth semilinear equation treated in [Christof et al., 2018] is weakly (sequentially) continuous on the considered spaces, see [Christof et al., 2018, Corollary 3.7], it always fulfills $\tilde{S}\left(u_{n}\right) \rightarrow \tilde{S}(u)$ whenever $u_{n} \rightarrow u$, anyway. However, the solution operator $S$ of the obstacle problem is not weakly (sequentially) continuous from $H^{-1}(\Omega)$ to $H_{0}^{1}(\Omega)$.

We collect some simple properties of the generalized derivatives.

Proposition 2.11. Let $T: X \rightarrow Y$ be a globally Lipschitz continuous map from a separable Banach space $X$ to a separable, reflexive Banach space $Y$.

(i) For all $x \in X$ it holds

$$
\partial_{B}^{s s} T(x) \subset \partial_{B}^{s w} T(x) \subset \partial_{B}^{w w} T(x) \quad \text { and } \quad \partial_{B}^{s s} T(x) \subset \partial_{B}^{w s} T(x) \subset \partial_{B}^{w w} T(x) .
$$

(ii) Let $x \in X$. Suppose there is a sequence $\left\{x_{n}\right\} \subset X$ with $x_{n} \rightarrow x$ in $X$ and a sequence $\left\{L_{n}\right\} \subset \mathcal{L}(X, Y)$ with $L_{n} \in \partial_{B}^{s s} T\left(x_{n}\right)$ for all $n \in \mathbb{N}$. Furthermore, assume that $L_{n} \stackrel{\text { SOT }}{\longrightarrow} L$ for some $L \in \mathcal{L}(X, Y)$. Then $L$ is in $\partial_{B}^{s s} T(x)$.

(iii) Let $x \in X$. Suppose there is a sequence $\left\{x_{n}\right\} \subset X$ with $x_{n} \rightarrow x$ in $X$ and a sequence $\left\{L_{n}\right\} \subset \mathcal{L}(X, Y)$ with $L_{n} \in \partial_{B}^{s w} T\left(x_{n}\right)$ for all $n \in \mathbb{N}$. Furthermore, assume that $L_{n} \stackrel{\text { WOT }}{\longrightarrow} L$ for some $L \in \mathcal{L}(X, Y)$. Then $L$ is in $\partial_{B}^{s w} T(x)$. 
Proof. The assertion in (i) follows easily by the relation between the respective topologies. (ii) can be found in [Christof et al., 2018, Proposition 3.4], one just hast to replace $L^{2}(\Omega)$ by an arbitrary separable Banach space $X$. We prove part (iii) similarly to [Christof et al., 2018, Proposition 3.4] with the obvious modifications: Since $L_{n} \in \partial_{B}^{s w} T\left(x_{n}\right)$, there are sequences $\left\{x_{m}^{(n)}\right\} \subset D_{T}$ with $x_{m}^{(n)} \rightarrow x_{n}$ as $m \rightarrow \infty$ and $T^{\prime}\left(x_{m}^{(n)}\right) \stackrel{\text { WOT }}{\longrightarrow} L_{n}$ as $m \rightarrow \infty$. Since $X$ is separable and since the properties of $Y$ imply that $Y^{\star}$ is separable as well, we can find sequences $\left\{h_{n}\right\}$ and $\left\{y_{n}^{\star}\right\}$ that are dense in $X$, respectively $Y^{\star}$. For all $n \in \mathbb{N}$ fix $m(n) \in \mathbb{N}$ with

$$
\begin{aligned}
\left|\left\langle y_{l}^{\star}, T^{\prime}\left(x_{m(n)}^{(n)}\right) h_{k}-L_{n} h_{k}\right\rangle\right| & <1 / n \quad \forall k, l=1, \ldots, n, \\
\left\|x_{m(n)}^{(n)}-x_{n}\right\| & \leq 1 / n .
\end{aligned}
$$

For fixed $h \in X, y^{\star} \in Y^{\star}$, and for all $n \in \mathbb{N}$ we define

$$
\begin{aligned}
& \bar{h}_{n}:=\arg \min \left\{\left\|h_{k}-h\right\|_{X}: 1 \leq k \leq n\right\}, \\
& \bar{y}_{n}^{\star}:=\arg \min \left\{\left\|y_{k}^{\star}-y^{\star}\right\|_{Y^{\star}}: 1 \leq k \leq n\right\} .
\end{aligned}
$$

These definitions imply that $\bar{h}_{n} \rightarrow h$ in $X$ and $\bar{y}_{n}^{\star} \rightarrow y^{\star}$ in $Y^{\star}$. We mention that all elements in $\partial_{B}^{w w} T(x)$ are bounded by the Lipschitz constant of $T$, see [Christof et al., 2018, Lemma 3.2(iii)]. In particular, $\left\|T^{\prime}\left(x_{m(n)}^{(n)}\right)-L_{n}\right\|_{\mathcal{L}(X, Y)}$ is bounded. This shows

$$
\begin{aligned}
& \mid\left\langle y^{\star},\right.\left.T^{\prime}\left(x_{m(n)}^{(n)}\right) h-L h\right\rangle \mid \\
& \leq \mid\left\langle\bar{y}_{n}^{\star}, T^{\prime}\left(x_{m(n)}^{(n)} \bar{h}_{n}-L_{n} \bar{h}_{n}\right\rangle|+|\left\langle\bar{y}_{n}^{\star}-y^{\star}, T\left(x_{m(n)}^{(n)}\right) \bar{h}_{n}-L_{n} \bar{h}_{n}\right\rangle\right| \\
& \quad+\left|\left\langle y^{\star},\left(T^{\prime}\left(x_{m(n)}^{(n)}\right)-L_{n}\right)\left(\bar{h}_{n}-h\right)\right\rangle\right|+\left|\left\langle y^{\star}, L_{n} h-L h\right\rangle\right| \\
& \leq 1 / n+\left\|\bar{y}_{n}^{\star}-y^{\star}\right\|_{Y^{\star}}\left\|T\left(x_{m(n)}^{(n)}\right)-L_{n}\right\|_{\mathcal{L}(X, Y)}\left\|\bar{h}_{n}\right\|_{X} \\
& \quad+\left\|y^{\star}\right\|_{Y^{\star}}\left\|T^{\prime}\left(x_{m(n)}^{(n)}\right)-L_{n}\right\|_{\mathcal{L}(X, Y)}\left\|\bar{h}_{n}-h\right\|_{X}+\left|\left\langle y^{\star}, L_{n} h-L h\right\rangle\right| \rightarrow 0
\end{aligned}
$$

as $n \rightarrow \infty$. Together with $x_{m(n)}^{(n)} \rightarrow x$, we obtain the desired $L \in \partial_{B}^{s w} T(x)$.

\section{Introduction to capacitary measures}

The goal of this paper is the characterization of generalized derivatives of the solution operator $S$. In Lemma 2.6, we have seen that $S^{\prime}(u ; \cdot)$ is of the form $L_{I(u)}$ for all differentiability points $u \in D_{S}$, see also (9). In the definitions of the generalized derivatives limits (in WOT or SOT) of such solution operators $L_{I(u)}$ appear, see Definition 2.10. Hence, we need to know which operators in $\mathcal{L}\left(H^{-1}(\Omega), H_{0}^{1}(\Omega)\right)$ can appear as limits (in WOT or SOT) of sequences of solution operators $L_{I(u)}$.

We will see that this question can be adequately answered by the concept of so-called capacitary measures. For the convenience of the reader, we will give a self-contained introduction. We suggest [Bucur, Buttazzo, 2005, Section 4.3], [Dal Maso, Garroni, 1994, Section 2] or [Dal Maso, Murat, 2004, Section 2.2] for further material. 
We also remark that Lemma 3.15, Theorem 3.16 and the second half of Theorem 3.9 are new results, while the remaining results can be found in the mentioned references or are easy corollaries of existing results in the literature.

Definition 3.1. Let $\mathcal{M}_{0}(\Omega)$ be the set of all Borel measures $\mu$ on $\Omega$ such that $\mu(B)=0$ for every Borel set $B \subset \Omega$ with $\operatorname{cap}(B)=0$ and such that $\mu$ is regular in the sense that $\mu(B)=\inf \left\{\mu(O): O\right.$ quasi-open, $\left.B \subset_{q} O\right\}$.

The set $\mathcal{M}_{0}(\Omega)$ is called the set of capacitary measures on $\Omega$. The name stems from the fact that, on the one hand, $\mu(B)=0$ for all Borel sets $B \subset \Omega$ with $\operatorname{cap}(B)=0$, and on the other hand, $\mu(B)=0$ for all $\mu \in \mathcal{M}_{0}(\Omega)$ implies that cap $(B)=0$, see [Bonnans, Shapiro, 2000, Lemma 6.55].

Recall that we work with Borel measurable representatives, that is, $v \in H_{0}^{1}(\Omega)$ is always assumed to be quasi-continuous and Borel measurable. Since $\mu \in \mathcal{M}_{0}(\Omega)$ is a Borel measure, $v$ is $\mu$-measurable. Further, for $p \in[1, \infty)$, we can define the integral

$$
\int_{\Omega}|v|^{p} \mathrm{~d} \mu \in[0, \infty]
$$

in the usual way. In the case that the integral is finite, we write $v \in L_{\mu}^{p}(\Omega)$. Note that this integral does not depend on the actual representative of $v$, since the quasi-continuous representatives differ only on sets of capacity zero whereas $\mu$ vanishes on sets of capacity zero.

For $\mu \in \mathcal{M}_{0}(\Omega)$ we consider the solution operator $L_{\mu}: H^{-1}(\Omega) \rightarrow H_{0}^{1}(\Omega)$ of the relaxed Dirichlet problem

$$
y \in H_{0}^{1}(\Omega): \quad-\Delta y+\mu y=f,
$$

that is, $L_{\mu}$ maps $f \in H^{-1}(\Omega)$ to the solution $y$ of

$$
\begin{aligned}
& y \in H_{0}^{1}(\Omega) \cap L_{\mu}^{2}(\Omega): \\
& \int_{\Omega} \nabla y \nabla z \mathrm{~d} x+\int_{\Omega} y z \mathrm{~d} \mu=\langle f, z\rangle_{H^{-1}(\Omega), H_{0}^{1}(\Omega)} \quad \forall z \in H_{0}^{1}(\Omega) \cap L_{\mu}^{2}(\Omega) .
\end{aligned}
$$

The solution to (10) exists and is unique, it can be identified with the Fréchet-Riesz representative of $f \in H^{-1}(\Omega) \subset\left(H_{0}^{1}(\Omega) \cap L_{\mu}^{2}(\Omega)\right)^{\prime}$ with respect to the scalar product $(y, z)=\int_{\Omega} \nabla y \nabla z \mathrm{~d} x+\int_{\Omega} y z \mathrm{~d} \mu$ on $H_{0}^{1}(\Omega) \cap L_{\mu}^{2}(\Omega)$. Indeed, $H_{0}^{1}(\Omega) \cap L_{\mu}^{2}(\Omega)$ is a Hilbert space, see [Buttazzo, Dal Maso, 1991, Proposition 2.1].

Let us motivate the notion of "relaxed Dirichlet problem". Let $O \subset \Omega$ be a quasi-open set. We define the measure $\infty_{\Omega \backslash O}$ via

$$
\infty_{\Omega \backslash O}(B)= \begin{cases}0, & \text { if } \operatorname{cap}(B \backslash O)=0, \\ +\infty, & \text { otherwise, }\end{cases}
$$

for all Borel sets $B \subset \Omega$. By definition, $\infty_{\Omega \backslash O}$ is a Borel measure and it is clear that $\infty_{\Omega \backslash O}$ vanishes on sets with zero capacity. The regularity of $\infty_{\Omega \backslash O}$ in the sense of Definition 3.1 is easy to check, see [Dal Maso, 1987, Remark 3.3]. Hence, $\infty_{\Omega \backslash O} \in \mathcal{M}_{0}(\Omega)$. From the 
definitions, it is easy to check that $v \in L_{\infty_{\Omega \backslash O}}^{2}(\Omega)$ if and only if $v=0$ q.e. on $\Omega \backslash O$ for all $v \in H_{0}^{1}(\Omega)$. That is, $H_{0}^{1}(\Omega) \cap L_{\infty_{\Omega \backslash O}}^{2}(\Omega)=H_{0}^{1}(O)$. Now, it is clear that the problem $(10)$ with $\mu=\infty_{\Omega \backslash O}$ is just a reformulation of the Dirichlet problem $-\Delta y=f$ in $H_{0}^{1}(O)^{\star}$. Therefore, the problems of class (10) with $\mu \in \mathcal{M}_{0}(\Omega)$ comprise the classical Dirichlet problem on open sets, but also more general problems.

Similarly, the problem

$$
y \in H_{0}^{1}(I(u)): \quad-\Delta y+\infty_{\Omega \backslash I(u)} y=f
$$

is an equivalent reformulation of (9). Therefore, the operators $L_{I(u)}$ (introduced in (9)) and $L_{\infty_{\Omega \backslash I(u)}}$ (from (10)) coincide. Thus, all possible Gâteaux derivatives of $S$ form a subset of $\left\{L_{\mu}: \mu \in \mathcal{M}_{0}(\Omega)\right\}$.

Next, we will describe how the set $\mathcal{M}_{0}(\Omega)$ can be equipped with a metric structure, rendering it a metric space with nice properties. We note that some references do not include the regularity condition from Definition 3.1 in the definition of $\mathcal{M}_{0}(\Omega)$. In the case that this regularity condition is dropped, one has to consider equivalence classes of capacitary measures in order to obtain a metric space. For a thorough discussion of this topic, we refer to [Dal Maso, 1987, Section 3].

Definition 3.2. Let $\left\{\mu_{n}\right\} \subset \mathcal{M}_{0}(\Omega)$. We say that the sequence $\left\{\mu_{n}\right\} \gamma$-converges to $\mu \in \mathcal{M}_{0}(\Omega)$ if and only if

$$
L_{\mu_{n}} \stackrel{\mathrm{WOT}}{\longrightarrow} L_{\mu} \text { in } \mathcal{L}\left(H^{-1}(\Omega), H_{0}^{1}(\Omega)\right) .
$$

If $\left\{\mu_{n}\right\} \gamma$-converges to $\mu$, we write $\mu_{n} \stackrel{\gamma}{\rightarrow} \mu$.

The name $\gamma$-convergence stems from the observation that this is closely related to the $\Gamma$-convergence of suitable functionals. To this end, we define $F_{\mu}: L^{2}(\Omega) \rightarrow[0, \infty]$ via

$$
F_{\mu}(u):= \begin{cases}\int_{\Omega}|\nabla u|^{2} \mathrm{~d} x+\int_{\Omega} u^{2} \mathrm{~d} \mu & \text { if } u \in H_{0}^{1}(\Omega) \cap L_{\mu}^{2}(\Omega) \\ +\infty & \text { else }\end{cases}
$$

for all $u \in L^{2}(\Omega)$ and $\mu \in \mathcal{M}_{0}(\Omega)$.

Definition 3.3. Let $\left\{\mu_{n}\right\} \subset \mathcal{M}_{0}(\Omega)$ and $\mu \in \mathcal{M}_{0}(\Omega)$ be given. We say that the functionals $F_{\mu_{n}} \Gamma$-converge towards $F_{\mu}$ in $L^{2}(\Omega)$ if and only if

$$
\begin{array}{ll}
\forall\left\{u_{n}\right\} \subset L^{2}(\Omega) \text { with } u_{n} \rightarrow u \text { in } L^{2}(\Omega): & F_{\mu}(u) \leq \liminf _{n \rightarrow \infty} F_{\mu_{n}}\left(u_{n}\right) \\
\exists\left\{u_{n}\right\} \subset L^{2}(\Omega) \text { with } u_{n} \rightarrow u \text { in } L^{2}(\Omega): & F_{\mu}(u)=\lim _{n \rightarrow \infty} F_{\mu_{n}}\left(u_{n}\right)
\end{array}
$$

hold for all $u \in L^{2}(\Omega)$. In this case, we write $F_{\mu_{n}} \stackrel{\Gamma}{\rightarrow} F_{\mu}$ in $L^{2}(\Omega)$.

The following lemma shows equivalent conditions for $\gamma$-convergence. 
Lemma 3.4. Let $\left\{\mu_{n}\right\} \subset \mathcal{M}_{0}(\Omega)$ and $\mu \in \mathcal{M}_{0}(\Omega)$ be given. Then, the following statements are equivalent:

(i) $\mu_{n} \stackrel{\gamma}{\rightarrow} \mu$.

(ii) $F_{\mu_{n}} \stackrel{\Gamma}{\rightarrow} F_{\mu}$ in $L^{2}(\Omega)$.

(iii) $L_{\mu_{n}} \stackrel{\text { SOT }}{\longrightarrow} L_{\mu}$ in $\mathcal{L}\left(L^{2}(\Omega), L^{2}(\Omega)\right)$.

(iv) $L_{\mu_{n}} \stackrel{\mathrm{WOT}}{\longrightarrow} L_{\mu}$ in $\mathcal{L}\left(L^{2}(\Omega), H_{0}^{1}(\Omega)\right)$.

(v) $L_{\mu_{n}}(1) \rightarrow L_{\mu}(1)$ in $L^{2}(\Omega)$.

(vi) $L_{\mu_{n}}(1) \rightarrow L_{\mu}(1)$ in $H_{0}^{1}(\Omega)$.

Proof. Let $\mu_{n} \stackrel{\gamma}{\rightarrow} \mu$. Then, for all $f \in H^{-1}(\Omega)$, in particular, for all $f \in L^{2}(\Omega)$, it holds $L_{\mu_{n}}(f) \rightarrow L_{\mu}(f)$ in $H_{0}^{1}(\Omega)$. Since $H_{0}^{1}(\Omega)$ is compactly embedded into $L^{2}(\Omega)$, it follows $L_{\mu_{n}}(f) \rightarrow L_{\mu}(f)$ in $L^{2}(\Omega)$, and thus (iii) holds.

Now, suppose that $L_{\mu_{n}} \stackrel{\text { SOT }}{\longrightarrow} L_{\mu}$ in $\mathcal{L}\left(L^{2}(\Omega), L^{2}(\Omega)\right)$. Let $f \in L^{2}(\Omega)$. By [Buttazzo, Dal Maso, 1991, (3.7)], there is a constant $c>0$, such that $\left\|L_{\mu_{n}}(f)\right\|_{H_{0}^{1}(\Omega)} \leq c\|f\|$ holds. Thus there is a subsequence $\left\{L_{\mu_{n_{k}}}\right\}$ that converges weakly in $H_{0}^{1}(\Omega)$. Hence $\left\{L_{\mu_{n_{k}}}(f)\right\}$ converges strongly in $L^{2}(\Omega)$ and the limit has to be $L_{\mu}(f)$. Thus, the whole sequence $\left\{L_{\mu_{n}}(f)\right\}$ converges weakly to $L_{\mu}(f)$ in $H_{0}^{1}(\Omega)$ and (iv) follows. The proof that (vi) follows from $(\mathrm{v})$ is also contained in this argument.

(vi) is an immediate consequence of (iv) and (v) follows from (vi) by the compact embedding of $H_{0}^{1}(\Omega)$ into $L^{2}(\Omega)$.

The equivalence of (vi) and (i) has been shown, in a more general setting, in [Dal Maso, Murat, 2004, Theorem 5.1].

The equivalence between (iii) and (ii) can be checked as in [Dal Maso, Mosco, 1987, Proposition 4.10].

Using the equivalence of $\mu_{n} \stackrel{\gamma}{\rightarrow} \mu$ and $\left\|L_{\mu_{n}}(1)-L_{\mu}(1)\right\|_{L^{2}(\Omega)} \rightarrow 0$, we can equip $\mathcal{M}_{0}(\Omega)$ with a metric.

Corollary 3.5. The $\gamma$-convergence on $\mathcal{M}_{0}(\Omega)$ is metrizable.

A different proof of this metrizability can be found in [Dal Maso, Mosco, 1987, Proposition 4.9].

The metric space $\mathcal{M}_{0}(\Omega)$ has many nice properties: it is complete (Lemma 3.6), the subset $\left\{\infty_{\Omega \backslash O}: O \subset \Omega\right.$ is quasi-open $\}$ is dense (Lemma 3.7) and $\mathcal{M}_{0}(\Omega)$ is compact (Theorem 3.8).

Lemma 3.6. The metric space $\mathcal{M}_{0}(\Omega)$ is complete.

For a proof, we refer to [Dal Maso, Mosco, 1987, Theorem 4.14] or [Dal Maso, Garroni, 1997, Theorem 4.5].

The next lemma shows that the measures $\infty_{C}$ with a quasi-closed set $C \subset \Omega$ represent a dense subclass of $\mathcal{M}_{0}(\Omega)$.

Lemma 3.7. Let $\mu$ be an element of $\mathcal{M}_{0}(\Omega)$. Then there is a sequence $\left\{O_{n}\right\}_{n \in \mathbb{N}} \subset \Omega$ of quasi-open sets such that $\infty_{\Omega \backslash O_{n}} \stackrel{\gamma}{\rightarrow} \mu$. 
A proof can be found in [Dal Maso, Mosco, 1987, Theorem 4.16] and a more constructive argument is given in [Dal Maso, Malusa, 1995].

The preceding lemma shows the connection between capacitary measures and shape optimization problem. Due to the fact that solutions of classical Dirichlet problems with varying (quasi-open) domains can converge to the solution of a relaxed Dirichlet problem with capacitary measures involved, an optimal domain in shape optimization might not exist, see e.g. [Bucur, Buttazzo, 2005, Section 4.2] or [Attouch et al., 2014, Section 5.8.4].

The next theorem shows the compactness of $\mathcal{M}_{0}(\Omega)$.

Theorem 3.8. Let $\left\{\mu_{n}\right\}$ be a sequence in $\mathcal{M}_{0}(\Omega)$. Then there exists a subsequence $\left\{\mu_{n_{k}}\right\}$ and a measure $\mu \in \mathcal{M}_{0}(\Omega)$ such that $\mu_{n_{k}} \stackrel{\gamma}{\rightarrow} \mu$.

For a proof, we refer to [Dal Maso, Mosco, 1987, Theorem 4.14]. Therein, one has to replace $\mathbb{R}^{n}$ by $\Omega$ to obtain the desired result.

Many properties of capacitary measures can be obtained by studying the so-called torsion function $w_{\mu}:=L_{\mu}(1)$. Indeed, we have already seen in Lemma 3.4 that it is sufficient to check the convergence $w_{\mu_{n}} \rightarrow w_{\mu}$ in $L^{2}(\Omega)$ of the torsion functions to obtain $\mu_{n} \stackrel{\gamma}{\rightarrow} \mu$. This implies in particular, that the measure $\mu$ is uniquely determined by its torsion function, see also [Dal Maso, Garroni, 1994, Proposition 3.4] and [Dal Maso, Garroni, 1997, Theorem 1.20].

Moreover, the next result shows that the torsion function $w$ associated with a quasi-open set $O \subset \Omega$ is positive on $O$, whereas the fine support of $1+\Delta w$ is $\Omega \backslash O$.

Theorem 3.9. Let $O \subset \Omega$ be quasi-open and set $w:=L_{O}(1)$. Then, $w \geq 0, O={ }_{q}\{w>$ $0\}$ and $1+\Delta w \in H^{-1}(\Omega)^{+}$with $\mathrm{f}-\operatorname{supp}(1+\Delta w)={ }_{q} \Omega \backslash O$.

Proof. It holds $w \geq 0$ by [Dal Maso, Garroni, 1994, Proposition 2.4]. The assertions $O={ }_{q}\{w>0\}$ and $1+\Delta w \in H^{-1}(\Omega)^{+}$are well known, see, e.g., [Velichkov, 2015, Proposition 3.4.26] and [Chipot, Dal Maso, 1992, Theorem 1].

It remains to check $C:={ }_{q} \mathrm{f}-\operatorname{supp}(1+\Delta w)={ }_{q} \Omega \backslash O$. Using the characterization of Lemma 2.5, we have

$$
\langle 1+\Delta w, v\rangle=0 \quad \Leftrightarrow \quad v=0 \text { q.e. on } C \quad \forall v \in H_{0}^{1}(\Omega)^{+} .
$$

Using $w=L_{O}(1)$, this directly implies that $C \subset_{q} \Omega \backslash O$. Next, we define $\hat{w}=L_{\Omega \backslash C}(1)$. Since $w \in H_{0}^{1}(O) \subset H_{0}^{1}(\Omega \backslash C)$, we have $\langle 1+\Delta \hat{w}, w\rangle=0$. Moreover, (13) implies $\langle 1+\Delta w, \hat{w}\rangle=0$. Using $\langle\Delta w, \hat{w}\rangle=\langle\Delta \hat{w}, w\rangle$, this implies

$$
\langle 1, \hat{w}-w\rangle=\int_{\Omega} \hat{w}-w \mathrm{~d} x=0 .
$$

Next, the comparison principle from [Dal Maso, Mosco, 1986, Theorem 2.10], see also [Dal Maso, Garroni, 1994, Proposition 2.5], implies $\hat{w} \geq w$ and, therefore, $\hat{w}=w$. Finally, the first part of the proof yields $\Omega \backslash C={ }_{q}\{\hat{w}>0\}={ }_{q}\{w>0\}=_{q} O$. Thus, $C={ }_{q}$ f-supp $(1+\Delta w)={ }_{q} \Omega \backslash O$. 
The next result shows that every capacitary measure can be approximated by Radon measures. Here, a Radon measure is a Borel measure which is finite on all compact subsets of $\Omega$.

Lemma 3.10. Let $\mu \in \mathcal{M}_{0}(\Omega)$. Then there exists an increasing sequence of Radon measures $\left\{\mu_{n}\right\}$ such that $\mu_{n} \stackrel{\gamma}{\rightarrow} \mu$.

Proof. Let $w_{0}:=L_{\Omega}(1)$ and for $\mu \in \mathcal{M}_{0}(\Omega)$ let $w:=L_{\mu}(1)$. In [Dal Maso, Garroni, 1994, Proposition 4.7], it is shown that for the sequence $\left\{w_{n}\right\}$ defined by

$$
w_{n}:=\left(1-\frac{1}{n}\right) w+\frac{1}{n} w_{0}
$$

the associated measures defined by

$$
\mu_{n}(B):= \begin{cases}\int_{B} \frac{\mathrm{d}\left(1+\Delta w_{n}\right)}{w_{n}}, & \text { if } \operatorname{cap}\left(B \cap\left\{w_{n}=0\right\}\right)=0 \\ +\infty, & \text { else }\end{cases}
$$

are Radon measures $\gamma$-converging to $\mu$. Thus, it remains to show the monotonicity of this sequence. Since $w_{0}>0$ by Theorem 3.9 , it holds $\mu_{n}(B)=\int_{B} \frac{\mathrm{d}\left(1+\Delta w_{n}\right)}{w_{n}}$ for all $n \in \mathbb{N}$ and for all Borel sets $B$. The representation

$$
\begin{aligned}
\mu_{n}(B) & =\int_{B} \frac{\mathrm{d}\left(1+\Delta w_{n}\right)}{w_{n}}=\int_{B} \frac{\mathrm{d}\left(1+\Delta\left((1-1 / n) w+1 / n w_{0}\right)\right)}{(1-1 / n) w+1 / n w_{0}} \\
& =\int_{B} \frac{\mathrm{d}(1-1 / n+(1-1 / n) \Delta w)}{(1-1 / n)\left(w+1 /(n-1) w_{0}\right)}=\int_{B} \frac{\mathrm{d}(1+\Delta w)}{w+1 /(n-1) w_{0}}
\end{aligned}
$$

shows that $\mu_{n} \leq \mu_{n+1} \leq \mu$ holds for all $n \in \mathbb{N}$.

The following lemma shows that the image of $L_{\mu}$ is dense in $H_{0}^{1}(\Omega) \cap L_{\mu}^{2}(\Omega)$.

Lemma 3.11. Let $\mu \in \mathcal{M}_{0}(\Omega)$ and let $y \in H_{0}^{1}(\Omega) \cap L_{\mu}^{2}(\Omega)$. Then there is a sequence

$$
\left\{y_{n}\right\} \subset\left\{L_{\mu}(f): f \in H^{-1}(\Omega)\right\}
$$

such that $y_{n} \rightarrow y$ in $H_{0}^{1}(\Omega) \cap L_{\mu}^{2}(\Omega)$.

Proof. For every $n \in \mathbb{N}$ let $y_{n} \in H_{0}^{1}(\Omega) \cap L_{\mu}^{2}(\Omega)$ be the solution of the problem

$$
\int_{\Omega} \nabla y_{n} \nabla v \mathrm{~d} x+\int_{\Omega} y_{n} v \mathrm{~d} \mu=-n \int_{\Omega}\left(y_{n}-y\right) v \mathrm{~d} x \quad \forall v \in H_{0}^{1}(\Omega) \cap L_{\mu}^{2}(\Omega) .
$$

We can write $y_{n}=L_{\mu}\left(-n\left(y_{n}-y\right)\right)$, thus $y_{n} \in\left\{L_{\mu}(f): f \in H^{-1}(\Omega)\right\}$. By [Dal Maso, Garroni, 1994, Proposition 3.1], it holds $y_{n} \rightarrow y$ in $H_{0}^{1}(\Omega) \cap L_{\mu}^{2}(\Omega)$ and the conclusion follows.

Lemma 3.12. Let $\mu \in \mathcal{M}_{0}(\Omega)$ and assume that $v \in H_{0}^{1}(\Omega) \cap L_{\mu}^{2}(\Omega)$. Then it holds $v=0$ q.e. on $\left\{w_{\mu}=0\right\}$ and $v \in H_{0}^{1}\left(\left\{w_{\mu}>0\right\}\right)$. 
Proof. By [Dal Maso, Garroni, 1994, Proposition 3.4], it holds $\mu(B)=+\infty$ for all Borel sets $B \subset \Omega$ with $\operatorname{cap}\left(B \cap\left\{w_{\mu}=0\right\}\right)>0$. Thus $v=0$ q.e. on $\left\{w_{\mu}=0\right\}$ for all $v$ in the image of $L_{\mu}$. By density of this set in $H_{0}^{1}(\Omega) \cap L_{\mu}^{2}(\Omega)$, see Lemma 3.11, it follows $v=0$ q.e. on $\left\{w_{\mu}=0\right\}$ for all $v \in H_{0}^{1}(\Omega) \cap L_{\mu}^{2}(\Omega)$.

It holds $w_{\mu} \geq 0$ on $\Omega$ by Theorem 3.9, therefore, each $v \in H_{0}^{1}(\Omega) \cap L_{\mu}^{2}(\Omega)$ is in $H_{0}^{1}\left(\left\{w_{\mu}>0\right\}\right)$.

The next result characterizes the completion of $H_{0}^{1}(\Omega) \cap L_{\mu}^{2}(\Omega)$ in $H_{0}^{1}(\Omega)$.

Lemma 3.13. Let $\mu \in \mathcal{M}_{0}(\Omega)$ be given. Then,

$$
{\overline{H_{0}^{1}}(\Omega) \cap L_{\mu}^{2}(\Omega)}_{H_{0}^{1}(\Omega)}^{=} H_{0}^{1}\left(\left\{w_{\mu}>0\right\}\right) .
$$

Moreover, for any $v \in H_{0}^{1}\left(\left\{w_{\mu}>0\right\}\right)^{+}$, there exists a sequence $\left\{v_{n}\right\}_{n \in \mathbb{N}} \subset H_{0}^{1}(\Omega) \cap L_{\mu}^{2}(\Omega)$ such that $0 \leq v_{n} \leq v$ q.e. on $\Omega$ for all $n \in \mathbb{N}$ and $v_{n} \rightarrow v$ in $H_{0}^{1}(\Omega)$.

Proof. We set $V:={\overline{H_{0}^{1}(\Omega) \cap L_{\mu}^{2}(\Omega)}}^{H_{0}^{1}(\Omega)}$. The inclusion $V \subset H_{0}^{1}\left(\left\{w_{\mu}>0\right\}\right)$ is clear from Lemma 3.12.

Then, it can be checked that $V$ is a closed lattice ideal in $H_{0}^{1}(\Omega)$, i.e., it is a closed subspace with the property that $v \in V, w \in H_{0}^{1}(\Omega)$ and $|w| \leq|v|$ imply $w \in V$. Hence, [Stollmann, 1993] implies that $V=H_{0}^{1}(\tilde{\Omega})$ for some quasi-open $\tilde{\Omega} \subset \Omega$. Thus,

$$
w_{\mu} \in V=H_{0}^{1}(\tilde{\Omega}) \subset H_{0}^{1}\left(\left\{w_{\mu}>0\right\}\right)
$$

and together with Theorem 3.9 we get $\tilde{\Omega}={ }_{q}\left\{w_{\mu}>0\right\}$. This shows $\overline{H_{0}^{1}(\Omega) \cap L_{\mu}^{2}(\Omega)}{ }^{H_{0}^{1}(\Omega)}=$ $V=H_{0}^{1}(\tilde{\Omega})=H_{0}^{1}\left(\left\{w_{\mu}>0\right\}\right)$.

The second assertion is clear since $w \mapsto \max (0, \min (w, v))$ is continuous on $H_{0}^{1}(\Omega)$.

Note that a similar assertion which, however, uses the so-called singular set of the measure $\mu$ can be found in [Buttazzo, Dal Maso, 1991, Lemma 2.6].

The next lemma shows that the solution operators associated with quasi-open sets form a (sequentially) closed set w.r.t. SOT.

Lemma 3.14. Let $\Omega_{n} \subset \Omega$ be a sequence of quasi-open sets such that $L_{\Omega_{n}}$ converges in the SOT towards some $L \in \mathcal{L}\left(H^{-1}(\Omega), H_{0}^{1}(\Omega)\right)$. Then, the limit satisfies $L=L_{\hat{\Omega}}$ for some quasi-open set $\hat{\Omega} \subset \Omega$.

Proof. From Lemma 3.6 we know that $L=L_{\mu}$ for some $\mu \in \mathcal{M}_{0}(\Omega)$. For given $f \in H^{-1}(\Omega)$, we set $v_{n}:=L_{\Omega_{n}} f$. Then, $v_{n} \rightarrow v:=L_{\mu} f$ in $H_{0}^{1}(\Omega)$ and this yields

$$
\int_{\Omega} v^{2} \mathrm{~d} \mu=\int_{\Omega}-|\nabla v|^{2}+f v \mathrm{~d} x=\lim _{n \rightarrow \infty} \int_{\Omega}-\left|\nabla v_{n}\right|^{2}+f v_{n} \mathrm{~d} x=0 .
$$

Hence, $\int_{\Omega} v^{2} \mathrm{~d} \mu=0$ for all $v$ in the range of $L_{\mu}$.

In order to check $L_{\mu}=L_{\hat{\Omega}}$ for some quasi-open set $\hat{\Omega} \subset \Omega$, we use the torsion function $w=L_{\mu}(1)$ and set $\hat{\Omega}:={ }_{q}\{w>0\}$. From $\int_{\Omega} w^{2} \mathrm{~d} \mu=0$ and $v \in H_{0}^{1}(\hat{\Omega})$ for $v \in H_{0}^{1}(\Omega) \cap L_{\mu}^{2}(\Omega)$ (see Lemma 3.12), it follows that $w=L_{\hat{\Omega}}(1)$. Thus, $L_{\hat{\Omega}}=L_{\mu}$ by [Dal Maso, Garroni, 1994, Proposition 3.4]. 
Note that we even have the following converse of Lemma 3.14. If $L_{\Omega_{n}} \stackrel{\text { WOT }}{\longrightarrow} L_{\Omega}$, then we already get $L_{\Omega_{n}} \stackrel{\text { SOT }}{\longrightarrow} L_{\Omega}$, see [Attouch et al., 2014, Proposition 5.8.6]. That is, the $\gamma$-limit of the sequence of quasi-open sets $\left\{\Omega_{n}\right\}$ is again a quasi-open set if and only if the solution operators converge in the strong operator topology.

Let us also mention that the $\gamma$-convergence of a sequence of quasi-open sets $\left\{\Omega_{n}\right\}$ to a quasi-open set $\tilde{\Omega}$, i.e., the convergence $L_{\Omega_{n}} \stackrel{\text { SOT }}{\longrightarrow} L_{\tilde{\Omega}}$, is equivalent to the convergence of the spaces $\left\{H_{0}^{1}\left(\Omega_{n}\right)\right\}$ to $H_{0}^{1}(\tilde{\Omega})$ in the sense of Mosco, see [Bucur, Buttazzo, 2005, Prop. 4.53, Remark 4.5.4]. This tool is also used in the derivation of a generalized gradient in [Rauls, Ulbrich, 2018].

As a last result in this section, we are going to study the convergence of a sum of two $\gamma$-convergent sequences. To this end, we need an auxiliary lemma.

Lemma 3.15. Let $\left\{u_{n}\right\},\left\{v_{n}\right\} \subset H_{0}^{1}(\Omega)$ be sequences with $u_{n} \rightarrow u$ in $H_{0}^{1}(\Omega)$ and $v_{n} \rightarrow u$ in $H_{0}^{1}(\Omega)$ for some $u \in H_{0}^{1}(\Omega)$. Then, $w_{n}:=\min \left(u_{n}, v_{n}\right)$ satisfies $w_{n} \rightarrow u$ in $H_{0}^{1}(\Omega)$ and

$$
\limsup _{n \rightarrow \infty}\left(\left\|w_{n}\right\|_{H_{0}^{1}(\Omega)}^{2}-\left\|v_{n}\right\|_{H_{0}^{1}(\Omega)}^{2}\right) \leq 0 .
$$

Proof. The weak convergence of $w_{n}$ follows from the weak sequential continuity of $\min (\cdot, \cdot)$ in $H_{0}^{1}(\Omega)$. To obtain the desired inequality, we check

$$
\begin{aligned}
\left\|w_{n}\right\|_{H_{0}^{1}(\Omega)}^{2}-\left\|v_{n}\right\|_{H_{0}^{1}(\Omega)}^{2} & =\left\|w_{n}-u_{n}\right\|_{H_{0}^{1}(\Omega)}^{2}-\left\|v_{n}-u_{n}\right\|_{H_{0}^{1}(\Omega)}^{2}+2\left(w_{n}-v_{n}, u_{n}\right)_{H_{0}^{1}(\Omega)} \\
& =-\left\|\max \left(0, v_{n}-u_{n}\right)\right\|_{H_{0}^{1}(\Omega)}^{2}+2\left(w_{n}-v_{n}, u_{n}\right)_{H_{0}^{1}(\Omega)} \\
& \leq 2\left(w_{n}-v_{n}, u_{n}\right)_{H_{0}^{1}(\Omega)} .
\end{aligned}
$$

Now, the claim follows from $w_{n}-v_{n} \rightarrow 0$ and $u_{n} \rightarrow u$ in $H_{0}^{1}(\Omega)$.

Theorem 3.16. Let $\left\{\mu_{n}\right\}$ be a sequence in $\mathcal{M}_{0}(\Omega)$ such that $\mu_{n} \stackrel{\gamma}{\rightarrow} \mu$ and let $\left\{C_{n}\right\}$ be a sequence of quasi-closed subsets of $\Omega$ such that $\infty_{C_{n}} \stackrel{\gamma}{\rightarrow} \infty_{C}$ for some quasi-closed set $C \subset \Omega$. Then, $\mu_{n}+\infty_{C_{n}} \stackrel{\gamma}{\rightarrow} \mu+\infty_{C}$.

Proof. We use the characterization of $\gamma$-convergence via the $\Gamma$-convergence of the functionals $F_{\mu_{n}+\infty_{C_{n}}}$. Therefore, we have to verify (12). Let $u \in L^{2}(\Omega)$ be given and consider an arbitrary sequence $\left\{u_{n}\right\} \subset L^{2}(\Omega)$ with $u_{n} \rightarrow u$ in $L^{2}(\Omega)$. We have to show

$$
F_{\mu+\infty_{C}}(u) \leq \liminf _{n \rightarrow \infty} F_{\mu_{n}+\infty_{C_{n}}}\left(u_{n}\right) .
$$

If the limes inferior is $+\infty$, there is nothing to show. Otherwise, we select a subsequence of $\left\{u_{n}\right\}$ (without relabeling), such that the limes inferior is actually a limit and such that $F_{\mu_{n}+\infty_{C_{n}}}\left(u_{n}\right)<+\infty$ for all $n$. This implies $u_{n} \in H_{0}^{1}(\Omega)$ as well as $\int_{\Omega} u_{n}^{2} \mathrm{~d} \infty_{C_{n}}<+\infty$, and these properties yield $u_{n} \in H_{0}^{1}\left(\Omega \backslash C_{n}\right)$. Consequently, we have

$$
F_{\infty_{C}}(u) \leq \liminf _{n \rightarrow \infty} F_{\infty_{C_{n}}}\left(u_{n}\right) \leq \liminf _{n \rightarrow \infty} F_{\mu_{n}+\infty_{C_{n}}}\left(u_{n}\right)<+\infty .
$$

Thus, $u \in H_{0}^{1}(\Omega \backslash C)$ and $\int_{\Omega} u^{2} \mathrm{~d} \infty_{C}=0$. Now, the desired inequality follows by

$$
F_{\mu+\infty_{C}}(u)=F_{\mu}(u) \leq \liminf _{n \rightarrow \infty} F_{\mu_{n}}\left(u_{n}\right)=\liminf _{n \rightarrow \infty} F_{\mu_{n}+\infty_{C_{n}}}\left(u_{n}\right),
$$


where we have used $F_{\mu_{n}} \stackrel{\Gamma}{\rightarrow} F_{\mu}$.

Further, we have to prove the existence of a sequence $\left\{w_{n}\right\} \subset L^{2}(\Omega)$ with $w_{n} \rightarrow u$ in $L^{2}(\Omega)$ and

$$
F_{\mu+\infty_{C}}(u)=\lim _{n \rightarrow \infty} F_{\mu_{n}+\infty_{C_{n}}}\left(w_{n}\right) .
$$

It is enough to consider the case $u \geq 0$, otherwise apply the following arguments to $u^{+}$ and $u^{-}$. If $F_{\mu+\infty_{C}}(u)=\infty$, there is nothing to show. Otherwise, we have $u \in H_{0}^{1}(\Omega \backslash C)$. From $F_{\mu_{n}} \stackrel{\Gamma}{\rightarrow} F_{\mu}$ and $F_{\infty_{C_{n}}} \stackrel{\Gamma}{\rightarrow} F_{\infty_{C}}$, we find sequences $\left\{v_{n}\right\},\left\{u_{n}\right\} \subset L^{2}(\Omega)$ with

$$
\begin{aligned}
& v_{n} \rightarrow u \text { in } L^{2}(\Omega) \quad \text { and } \quad F_{\mu_{n}}\left(v_{n}\right) \rightarrow F_{\mu}(u), \\
& u_{n} \rightarrow u \text { in } L^{2}(\Omega) \quad \text { and } \quad F_{\infty_{C_{n}}}\left(u_{n}\right) \rightarrow F_{\infty_{C}}(u) .
\end{aligned}
$$

W.l.o.g., we can assume $v_{n}, u_{n} \geq 0$ (otherwise, replace $v_{n}$ by $\max \left(v_{n}, 0\right)$ and $u_{n}$ by $\left.\max \left(u_{n}, 0\right)\right)$. We easily infer $v_{n} \rightarrow u$ in $H_{0}^{1}(\Omega)$ and $u_{n} \rightarrow u$ in $H_{0}^{1}(\Omega)$. We define $w_{n}=\min \left(u_{n}, v_{n}\right)$ and already get $w_{n} \rightarrow u$ in $L^{2}(\Omega)$. To obtain the convergence of the function values, we use $w_{n}=0$ q.e. on $C_{n}$ to obtain

$$
\begin{aligned}
F_{\mu_{n}+\infty_{C_{n}}}\left(w_{n}\right) & =F_{\mu_{n}}\left(w_{n}\right)=\int_{\Omega}\left|\nabla w_{n}\right|^{2} \mathrm{~d} x+\int_{\Omega} w_{n}^{2} \mathrm{~d} \mu_{n} \\
& \leq \int_{\Omega}\left|\nabla w_{n}\right|^{2} \mathrm{~d} x+\int_{\Omega} v_{n}^{2} \mathrm{~d} \mu_{n}=F_{\mu_{n}}\left(v_{n}\right)+\left(\left\|w_{n}\right\|_{H_{0}^{1}(\Omega)}^{2}-\left\|v_{n}\right\|_{H_{0}^{1}(\Omega)}^{2}\right) .
\end{aligned}
$$

Now, by using Lemma 3.15 and $u \in H_{0}^{1}(\Omega \backslash C)$ we obtain

$$
\begin{aligned}
F_{\mu+\infty_{C}}(u) & \leq \liminf _{n \rightarrow \infty} F_{\mu_{n}+\infty_{C_{n}}}\left(w_{n}\right) \\
& \leq \limsup _{n \rightarrow \infty} F_{\mu_{n}+\infty_{C_{n}}}\left(w_{n}\right) \leq \limsup _{n \rightarrow \infty} F_{\mu_{n}}\left(v_{n}\right)=F_{\mu}(u)=F_{\mu+\infty_{C}}(u) .
\end{aligned}
$$

Thus, $F_{\mu+\infty_{C}}(u)=\lim _{n \rightarrow \infty} F_{\mu_{n}+\infty_{C_{n}}}\left(w_{n}\right)$. This finishes the proof of $F_{\mu_{n}+\infty_{C_{n}}}\left(w_{n}\right) \stackrel{\Gamma}{\rightarrow}$ $F_{\mu+\infty}(u)$.

\section{Generalized derivatives involving the SOT}

In this section, we are going to characterize the generalized derivatives of the obstacle problem which involve the SOT.

Therefore, as a technique, we frequently use the argument that if $\hat{\Omega} \subset \Omega$ is quasi-open and if $v \in H_{0}^{1}(\hat{\Omega})$, then this implies $v=L_{\hat{\Omega}}(-\Delta v)$.

As a first result, we give an upper estimate for $\partial_{B}^{w s} S(u)$.

Lemma 4.1. Let us assume that $L \in \partial_{B}^{w s} S(u)$. Then, there exists a quasi-open set $\hat{\Omega} \subset \Omega$ with $\operatorname{cap}\left(\hat{\Omega} \cap A_{s}(u)\right)=0$ and $L=L_{\hat{\Omega}}$.

Proof. By definition, there is a sequence $\left\{u_{n}\right\} \subset D_{S}$ such that $u_{n} \rightarrow u$ in $H^{-1}(\Omega)$, $S\left(u_{n}\right) \rightarrow S(u)$ in $H_{0}^{1}(\Omega)$ and $S^{\prime}\left(u_{n}\right) \stackrel{\text { SOT }}{\longrightarrow} L$. By the characterization of differentiability points of $S$, we have $S^{\prime}\left(u_{n}\right)=L_{I\left(u_{n}\right)}$. From Lemma 3.14, we already know that $L=L_{\hat{\Omega}}$ for some quasi-open set $\hat{\Omega} \subset \Omega$. It remains to $\operatorname{check} \operatorname{cap}\left(\hat{\Omega} \cap A_{s}(u)\right)=0$. 
From Theorem 3.9 we infer the existence of $v \in H_{0}^{1}(\Omega)^{+}$with $\{v>0\}={ }_{q} \hat{\Omega}$. In particular, $v \in H_{0}^{1}(\hat{\Omega})$ and this yields that $v=L_{\hat{\Omega}}(-\Delta v)$ is the strong limit of $v_{n}:=$ $S^{\prime}\left(u_{n}\right)(-\Delta v)$. By the properties of $S^{\prime}\left(u_{n}\right)$, we have $v_{n}=0$ q.e. on $A_{s}\left(u_{n}\right)$. Thus,

$$
\left\langle\xi_{n},\left|v_{n}\right|\right\rangle=0,
$$

where $\xi_{n}:=-\Delta S\left(u_{n}\right)-u_{n}$. From $\xi_{n} \rightarrow \xi:=-\Delta S(u)-u$ we infer

$$
\langle\xi,|v|\rangle=0 .
$$

Thus, $v=0$ q.e. on $A_{s}(u)$. Hence, $\operatorname{cap}\left(\hat{\Omega} \cap A_{s}(u)\right)=\operatorname{cap}\left(\{v \neq 0\} \cap A_{s}(u)\right)=0$.

Before we can give a precise characterization of $\partial_{B}^{w s} S(u)$ and $\partial_{B}^{s s} S(u)$, we need an auxiliary lemma.

Lemma 4.2. Let a sequence $u_{n} \rightarrow u$ in $H^{-1}(\Omega)$ be given. Then, for every $v \in H_{0}^{1}(I(u))$ with $0 \leq v \leq 1$, there exists a sequence $\left\{v_{n}\right\}$ with $v_{n} \in H_{0}^{1}\left(I\left(u_{n}\right)\right)$ and $v_{n} \rightarrow v$ in $H_{0}^{1}(\Omega)$.

Proof. We set $y=S(u)$ and $y_{n}=S\left(u_{n}\right)$. Let $t_{n}:=\sup _{m=n, \ldots, \infty}\left\|y_{m}-y\right\|_{H_{0}^{1}(\Omega)}^{1 / 2}$. Then, $\left\{t_{n}\right\}$ is a decreasing sequence of nonnegative numbers with $t_{n} \geq\left\|y_{n}-y\right\|_{H_{0}^{1}(\Omega)}^{1 / 2}$ and $t_{n} \searrow 0$. We have $\{y>\psi\}={ }_{q} \bigcup_{n=1}^{\infty}\left\{y>\psi+t_{n}\right\}$. Since the sets on the right-hand side are quasi-open and increasing in $n$, we can apply Lemma 2.3. This yields a sequence $\left\{\tilde{v}_{n}\right\} \subset H_{0}^{1}(\Omega)$ with $\tilde{v}_{n} \rightarrow v$ in $H_{0}^{1}(\Omega), 0 \leq \tilde{v}_{n} \leq 1$ and $\tilde{v}_{n}=0$ q.e. on $\left\{y \leq \psi+t_{n}\right\}$.

Next, we have

$$
\operatorname{cap}\left(\left\{y_{n}=\psi\right\} \cap\left\{y>\psi+t_{n}\right\}\right) \leq \operatorname{cap}\left(\left\{\left|y_{n}-y\right|>t_{n}\right\}\right) \leq t_{n}^{-2}\left\|y_{n}-y\right\|_{H_{0}^{1}(\Omega)}^{2} \rightarrow 0 .
$$

Thus, there exists $w_{n} \in H_{0}^{1}(\Omega)$ with $w_{n} \rightarrow 0$ in $H_{0}^{1}(\Omega), 0 \leq w_{n} \leq 1$ and $w_{n}=1$ q.e. on $\left\{\left|y_{n}-y\right|>t_{n}\right\}$. We set $v_{n}:=\max \left(\tilde{v}_{n}-w_{n}, 0\right)$. By construction, $v_{n} \rightarrow v$ and $v_{n}=0$ q.e. on $\left\{y_{n}=\psi\right\}$, i.e., $v_{n} \in H_{0}^{1}\left(I\left(u_{n}\right)\right)$.

Next, we give a characterization of $\partial_{B}^{s s} S(u)$.

Theorem 4.3. Let $u \in H^{-1}(\Omega)$ be given. Then,

$$
\partial_{B}^{s s} S(u)=\left\{L_{\hat{\Omega}} \mid \hat{\Omega} \text { is quasi-open and } I(u) \subset_{q} \hat{\Omega} \subset_{q} \Omega \backslash A_{s}(u)\right\} .
$$

Proof. "C": Let $L \in \partial_{B}^{s s} S(u)$ be given. By definition, $S^{\prime}\left(u_{n}\right) \stackrel{\text { SOT }}{\longrightarrow} L$ for some sequence $\left\{u_{n}\right\} \subset D_{S}$ with $u_{n} \rightarrow u$. From Lemma 4.1 and $L \in \partial_{B}^{s s} S(u) \subset \partial_{B}^{w s} S(u)$, we already have $L=L_{\hat{\Omega}}$ for some quasi-open $\hat{\Omega} \subset_{q} \Omega \backslash A_{s}(u)$. It remains to check $I(u) \subset_{q} \hat{\Omega}$.

By Theorem 3.9, there is a function $v \in H_{0}^{1}(\Omega)$ with $0 \leq v \leq 1$ and $I(u)=_{q}\{v>0\}$. From Lemma 4.2, we get a sequence $\left\{v_{n}\right\}$ with $v_{n} \rightarrow v$ and $v_{n} \in H_{0}^{1}\left(I\left(u_{n}\right)\right)$. Together with Lemma 2.9, we find

$$
v=\lim _{n \rightarrow \infty} v_{n}=\lim _{n \rightarrow \infty} S^{\prime}\left(u_{n}\right)\left(-\Delta v_{n}\right)=L_{\hat{\Omega}}(-\Delta v) .
$$

This gives $I(u)={ }_{q}\{v>0\} \subset_{q} \hat{\Omega}$. 
" $\supset$ ": Let $\hat{\Omega}$ be given as in the formulation of the theorem. From Theorem 3.9, we get a function $v \in H_{0}^{1}(\Omega)^{+}$with $\{v>0\}={ }_{q} \hat{\Omega}$. Similarly, Theorem 3.9 gives $\lambda \in H^{-1}(\Omega)^{+}$ with $\mathrm{f}-\operatorname{supp}(\lambda)={ }_{q} \Omega \backslash \hat{\Omega}$. We define $u_{n}:=u-(\Delta v+\lambda) / n$. Let us check that $y_{n}:=y+v / n$ satisfies $y_{n}=S\left(u_{n}\right)$. From $v \geq 0$, we infer $y_{n} \in K$. Further, for arbitrary $z \in K$ we have

$$
\begin{aligned}
\left\langle-\Delta y_{n}-u_{n}, z-y_{n}\right\rangle & =\left\langle-\Delta y-\frac{1}{n} \Delta v-u+\frac{1}{n} \Delta v+\frac{1}{n} \lambda, z-y-\frac{1}{n} v\right\rangle \\
& =\langle-\Delta y-u, z-y\rangle+\left\langle-\Delta y-u,-\frac{1}{n} v\right\rangle+\left\langle\frac{1}{n} \lambda, z-y-\frac{1}{n} v\right\rangle \\
& \geq 0+0+0 .
\end{aligned}
$$

The second term is zero due to $\xi=-\Delta y-u, f-\operatorname{supp}(\xi)={ }_{q} A_{s}(u)$ and $v=0$ on $\Omega \backslash \hat{\Omega} \supset_{q} A_{s}(u)$. Similarly, the third term is non-negative since f-supp $(\lambda)={ }_{q} \Omega \backslash \hat{\Omega}$ and $z \geq \psi=y+v / n$ on $\Omega \backslash \hat{\Omega}$. Hence, $y_{n}=S\left(u_{n}\right)$ and $\xi_{n}:=-\Delta y_{n}-u_{n}=\xi+\lambda / n$. Thus, $I\left(u_{n}\right)=_{q} \hat{\Omega}={ }_{q} \Omega \backslash A_{s}\left(u_{n}\right)$, i.e., $u_{n} \in D_{S}$. Finally, $S^{\prime}\left(u_{n}\right)=L_{\hat{\Omega}}$ and $u_{n} \rightarrow u$ ensure $L_{\hat{\Omega}} \in \partial_{B}^{s s} S(u)$.

We can also give a characterization of $\partial_{B}^{w s} S(u)$.

Theorem 4.4. Let $u \in H^{-1}(\Omega)$ be given. Then,

$$
\partial_{B}^{w s} S(u)=\left\{L_{\hat{\Omega}} \mid \hat{\Omega} \text { is quasi-open and } I(u) \subset_{q} \hat{\Omega} \subset_{q} \Omega \backslash A_{s}(u)\right\} .
$$

Proof. "C": Let $L \in \partial_{B}^{w s} S(u)$ be given. By definition, $S^{\prime}\left(u_{n}\right) \stackrel{\text { SOT }}{\longrightarrow} L$ for some sequence $\left\{u_{n}\right\} \subset D_{S}$ with $u_{n} \rightarrow u$ and $S\left(u_{n}\right) \rightarrow S(u)$. From Lemma 4.1, we already have $L=L_{\hat{\Omega}}$ for some quasi-open $\hat{\Omega} \subset_{q} \Omega \backslash A_{s}(u)$. It remains to check $I(u) \subset_{q} \hat{\Omega}$.

We set $w=L_{\hat{\Omega}} 1$ and $w_{n}=S^{\prime}\left(u_{n}\right) 1=L_{I\left(u_{n}\right)} 1$. From Theorem 3.9, we find $1+\Delta w_{n} \geq 0$ and f-supp $\left(1+\Delta w_{n}\right)={ }_{q} A\left(u_{n}\right)$. Since $y_{n}:=S\left(u_{n}\right)=\psi$ q.e. on $A\left(u_{n}\right)$ and since $y_{n}$ and $\psi$ are assumed to be Borel measurable, this gives

$$
\int_{\Omega}\left(y_{n}-\psi\right) \mathrm{d}\left(1+\Delta w_{n}\right)=0 .
$$

In the next few lines, we need to work with a capacity on all of $\mathbb{R}^{d}$. This can be defined as in [Dal Maso, 1983, Section 1]. The function $y-\psi$ is non-negative and quasi lower-semicontinuous. Moreover, if we extend this function by 0 , it is quasi lowersemicontinuous on all of $\mathbb{R}^{d}$. Now, [Dal Maso, 1983, Lemma 1.5] implies the existence of an increasing sequence $\left\{z_{m}\right\}_{m \in \mathbb{N}} \subset H^{1}\left(\mathbb{R}^{d}\right)$ with $0 \leq z_{m}$ and $z_{m} \nearrow y-\psi$ pointwise q.e. on $\mathbb{R}^{d}$. From $y-\psi=0$ on $\mathbb{R}^{d} \backslash \Omega$, we have $z_{m}=0$ q.e. on $\mathbb{R}^{d} \backslash \Omega$. Thus, $z_{m} \in H_{0}^{1}(\Omega)$, see [Heinonen et al., 1993, Theorem 4.5]. This yields

$$
\int_{\Omega}\left(z_{m}-y+y_{n}\right) \mathrm{d}\left(1+\Delta w_{n}\right) \leq \int_{\Omega}\left(y_{n}-\psi\right) \mathrm{d}\left(1+\Delta w_{n}\right)=0 .
$$

From $y_{n} \rightarrow y$ in $H_{0}^{1}(\Omega)$ and $w_{n} \rightarrow w$ in $H_{0}^{1}(\Omega)$, we infer

$$
0 \leq \int_{\Omega} z_{m} \mathrm{~d}(1+\Delta w)=\lim _{n \rightarrow \infty} \int_{\Omega}\left(z_{m}-y+y_{n}\right) \mathrm{d}\left(1+\Delta w_{n}\right) \leq 0 .
$$


Hence,

$$
\int_{\Omega} z_{m} \mathrm{~d}(1+\Delta w)=0
$$

Finally, $\left\{z_{m}\right\}$ converges monotonically pointwise q.e. to $y-\psi$. The monotone convergence theorem implies

$$
\int_{\Omega}(y-\psi) \mathrm{d}(1+\Delta w)=\lim _{m \rightarrow \infty} \int_{\Omega} z_{m} \mathrm{~d}(1+\Delta w)=0 .
$$

Therefore, $y-\psi=0$ q.e. on $\mathrm{f}-\operatorname{supp}(1+\Delta w)={ }_{q} \Omega \backslash \hat{\Omega}$. Hence, $\Omega \backslash \hat{\Omega} \subset_{q} A(u)$ and this yields the desired $I(u) \subset_{q} \hat{\Omega}$.

"つ": This follows from $\partial_{B}^{w s} S(u) \supset \partial_{B}^{s s} S(u)$ and Theorem 4.3.

Theorems 4.3 and 4.4 show that $\partial_{B}^{w s} S(u)=\partial_{B}^{s s} S(u)$ for all $u \in H^{-1}(\Omega)$ without any regularity assumptions on the data.

\section{The strong-weak generalized derivative}

In this section, we investigate $\partial_{B}^{s w} S(u)$. Since this generalized differential involves the WOT for the convergence of the derivatives, we expect that the resulting set is significantly larger than $\partial_{B}^{s s} S(u)$. In fact, we will see that capacitary measures enter the stage. As a first result, we prove an upper bound.

Lemma 5.1. Let $u \in H^{-1}(\Omega)$ be given. Then,

$$
\partial_{B}^{s w} S(u) \subset\left\{L_{\mu} \mid \mu \in \mathcal{M}_{0}(\Omega), \mu(I(u))=0 \text { and } \mu=+\infty \text { on } A_{s}(u)\right\} .
$$

Here, $\mu=+\infty$ on $A_{s}(u)$ is to be understood as

$$
\forall v \in H_{0}^{1}(\Omega) \cap L_{\mu}^{2}(\Omega): \quad v=0 \text { q.e. on } A_{s}(u) .
$$

Proof. Let $L \in \partial_{B}^{s w} S(u)$ be given. By definition, there is a sequence $\left\{u_{n}\right\} \subset D_{S}$ with $u_{n} \rightarrow u$ in $H^{-1}(\Omega)$ and $S^{\prime}\left(u_{n}\right) \stackrel{\text { WOT }}{\longrightarrow} L$. From Lemma 3.6 we obtain $L=L_{\mu}$ for some $\mu \in \mathcal{M}_{0}(\Omega)$.

First, we show $\mu=+\infty$ on $A_{s}(u)$. Let $f \in H^{-1}(\Omega)$ be given. Then, $v_{n}:=S^{\prime}\left(u_{n}\right) f \rightarrow$ $L_{\mu} f=: v$ and $\left|v_{n}\right| \rightarrow|v|$ in $H_{0}^{1}(\Omega)$. For $\xi_{n}:=-\Delta S\left(u_{n}\right)-u_{n}$ and $\xi:=-\Delta S(u)-u$ we have $\xi_{n} \rightarrow \xi$ in $H^{-1}(\Omega)$. It holds $\left|v_{n}\right|=0$ q.e. on $\mathrm{f}-\operatorname{supp}\left(\xi_{n}\right)={ }_{q} A_{s}\left(u_{n}\right)$. This implies

$$
0=\lim _{n \rightarrow \infty}\left\langle\xi_{n},\left|v_{n}\right|\right\rangle=\langle\xi,|v|\rangle .
$$

Hence, $|v|=0$ q.e. on f-supp $(\xi)={ }_{q} A_{s}(u)$. Since the range of $L_{\mu}$ is dense in $H_{0}^{1}(\Omega) \cap L_{\mu}^{2}(\Omega)$, see Lemma 3.11, we have $\mu=+\infty$ on $A_{s}(u)$.

It remains to show $\mu(I(u))=0$. Let $v \in H_{0}^{1}(I(u))$ with $0 \leq v \leq 1$ and $\{v>0\}={ }_{q} I(u)$ be given, see Theorem 3.9. By Lemma 4.2, there exists a sequence $\left\{v_{n}\right\}$ with $v_{n} \rightarrow v$ in $H_{0}^{1}(\Omega)$ and $v_{n} \in H_{0}^{1}\left(I\left(u_{n}\right)\right)$. Therefore, $v_{n}=S^{\prime}\left(u_{n}\right)\left(-\Delta v_{n}\right)$. Since $-\Delta v_{n} \rightarrow-\Delta v$ in 
$H^{-1}(\Omega)$, Lemma 2.9 (ii) implies $v_{n}=S^{\prime}\left(u_{n}\right)\left(-\Delta v_{n}\right) \rightarrow L_{\mu}(-\Delta v)$. Hence, $v=L_{\mu}(-\Delta v)$ and therefore, $v \in L_{\mu}^{2}(\Omega)$. Testing the associated weak formulation with $v$, we infer

$$
\int_{\Omega}|\nabla v|^{2} \mathrm{~d} x+\int_{\Omega} v^{2} \mathrm{~d} \mu=\langle-\Delta v, v\rangle=\int_{\Omega}|\nabla v|^{2} \mathrm{~d} x .
$$

Hence, $\int_{\Omega} v^{2} \mathrm{~d} \mu=0$ and this means $v=0 \mu$-a.e. on $\Omega$. Since $v>0$ q.e. on $I(u)$ and since $\mu$ does not charge polar sets, we have $v>0 \mu$-a.e. on $I(u)$. This implies $\mu(I(u))=0$.

To illustrate the meaning of $\mu=+\infty$ on $A_{s}(u)$, we give some equivalent reformulations.

Lemma 5.2. Let $u \in H^{-1}(\Omega)$ and $\mu \in \mathcal{M}_{0}(\Omega)$ be given. Then, the following assertions are equivalent.

(i) $\mu=+\infty$ on $A_{s}(u)$ in the sense of (15).

(ii) $\forall v \in H_{0}^{1}\left(\left\{w_{\mu}>0\right\}\right): \quad v=0$ q.e. on $A_{s}(u)$.

(iii) $w_{\mu}=0$ q.e. on $A_{s}(u)$.

(iv) $\mu \geq \infty_{A_{s}(u)}$.

Proof. The equivalence between (i) and (ii) follows from Lemma 3.13. From Lemma 3.12, we get that (ii) and (iii) are equivalent.

Let us assume that (iii) holds. By [Dal Maso, Garroni, 1994, Proposition 3.4], it holds $\mu(B)=+\infty$ for all Borel sets $B \subset \Omega$ with $\operatorname{cap}\left(B \cap\left\{w_{\mu}=0\right\}\right)>0$ and this gives (iv).

Finally, (iv) implies (iii) by the comparison principle [Dal Maso, Mosco, 1986, Theorem 2.10].

Note that if $u$ is a differentiability point of $S$, then the right-hand side in (14) reduces to $\left\{S^{\prime}(u)\right\}$ and equality holds.

In the general case, the reverse inclusion in (14) is much harder to obtain, and we will prove it under some regularity assumption on $\psi$. However, in the very simple and artificial case that the entire set $\Omega$ is biactive, i.e., $A(u)={ }_{q} \Omega$ and $A_{s}(u)={ }_{q} \emptyset$, the equality in (14) just follows from the density result in Lemma 3.7.

Corollary 5.3. Let $u \in H^{-1}(\Omega)$ be given such that $A(u)={ }_{q} \Omega$ and $A_{s}(u)={ }_{q} \emptyset$. Then,

$$
\partial_{B}^{s w} S(u)=\left\{L_{\mu} \mid \mu \in \mathcal{M}_{0}(\Omega)\right\} .
$$

In particular, (14) holds with equality.

Proof. The inclusion " $\subset$ " is established in Lemma 5.1 and it remains to check " $\supset$ ". From Theorem 4.3, we have

$$
\partial_{B}^{s s} S(u)=\left\{L_{\hat{\Omega}} \mid \hat{\Omega} \subset \Omega \text { is quasi-open }\right\} \subset \partial_{B}^{s w} S(u) .
$$

Since the closure of the left-hand side w.r.t. WOT is $\left\{L_{\mu} \mid \mu \in \mathcal{M}_{0}(\Omega)\right\}$, see Lemma 3.7, and since $\partial_{B}^{s w} S(u)$ is closed in WOT, see Proposition 2.11, this yields the claim. 
The verification of the reverse inclusion in (14) in the general case is much more delicate. The reason is that the density result Lemma 3.7 is typically proved in a rather abstract way, i.e., it is not easy to obtain the approximating sequence of quasi-open sets $O_{n}$. We are going to use the explicit construction from [Dal Maso, Malusa, 1995]. This, however, needs that $A\left(u_{n}\right)$ contains an open neighborhood of $A(u)$ and, therefore, we have to assume some regularity of $y$ and $\psi$. We give some preparatory lemmas.

Lemma 5.4. Let $u \in H^{-1}(\Omega)$ be given and define $y:=S(u)$. We assume that $y \in C_{0}(\Omega)$, $\psi \in C(\bar{\Omega}) \cap H^{1}(\Omega)$. Further, we assume that $\psi \in H_{0}^{1}(\Omega)$ or $\psi<0$ on $\partial \Omega$.

Then, there exists a sequence $\left\{u_{n}\right\} \subset H^{-1}(\Omega)$ such that $u_{n} \rightarrow u$ in $H^{-1}(\Omega), y_{n}:=S\left(u_{n}\right)$ satisfies $y_{n}=\psi$ on $\{y<\psi+1 / n\}$ and $\xi=-\Delta y-u=-\Delta y_{n}-u_{n}$. In particular, $\{y<\psi+1 / n\}$ is an open neighborhood of $\{y=\psi\}$.

Proof. Our strategy is to define $y_{n}$ with the desired properties and to verify afterwards that $y_{n}$ solves the obstacle problem with right-hand side $u_{n}:=-\Delta y_{n}-\xi$.

In the case that $\psi \in H_{0}^{1}(\Omega)$, we define $y_{n}:=\max (y-1 / n, \psi)$. It is immediate that $y_{n} \in H_{0}^{1}(\Omega), y_{n} \rightarrow y$ in $H_{0}^{1}(\Omega)$ and $y_{n}=\psi$ on $\{y<\psi+1 / n\}$.

In the case that $\psi<0$ on $\partial \Omega$, we have $\psi \leq c$ on $\partial \Omega$ for some constant $c<0$. From $y=0$ on $\partial \Omega$, we find that the set $\{y=\psi\}$ has a positive distance to the boundary of $\Omega$. Thus, there exists a function $\varphi \in C_{c}^{\infty}(\Omega)$ with $0 \leq \varphi \leq 1$ and $\varphi=1$ on $\{y=\psi\}$. Now, we set $y_{n}:=\max (y-\varphi / n, \psi)$. Again, we find $y_{n} \in H_{0}^{1}(\Omega), y_{n} \rightarrow y$ in $H_{0}^{1}(\Omega)$ and $y_{n}=\psi$ on $\{y<\psi+1 / n\}$.

Finally, we define $u_{n}:=-\Delta y_{n}-\xi$. It is immediate that $u_{n} \rightarrow u$ in $H^{-1}(\Omega)$ and we have to check that $y_{n}=S\left(u_{n}\right)$. The property $y_{n} \in K$ is immediate from the definition. From f-supp $(\xi)={ }_{q} A_{s}(u) \subset_{q}\{y=\psi\} \subset\left\{y_{n}=\psi\right\}$, we infer f-supp $(\xi) \subset_{q}\left\{z \geq y_{n}\right\}$ for all $z \in K$. Hence,

$$
\left\langle-\Delta y_{n}-u_{n}, z-y_{n}\right\rangle=\left\langle\xi, z-y_{n}\right\rangle=\int_{\Omega}\left(z-y_{n}\right) \mathrm{d} \xi \geq 0 .
$$

This shows that $y_{n}=S\left(u_{n}\right)$.

The next result shows that we can approximate solution operators associated to Radon measures.

Lemma 5.5. Let $u \in H^{-1}(\Omega)$ be given such that the assumptions of Lemma 5.4 are satisfied. Then, for every Radon measure $\mu \in \mathcal{M}_{0}(\Omega)$ with $\mu(I(u))=0$, the measure $\lambda=\mu+\infty_{A_{s}(u)}$ satisfies

$$
L_{\lambda} \in \partial_{B}^{s w} S(u) .
$$

Proof. Let $\mu$ be a given Radon measure as in the formulation of the lemma. We can use the construction of [Dal Maso, Malusa, 1995, Theorem 2.5] to obtain a sequence $\left\{E_{m}\right\}$ of compact subsets of $\Omega$ with the property that each $E_{m}$ is contained in $\operatorname{supp}(\mu)+B_{1 / m}$ and $\infty_{E_{m}} \stackrel{\gamma}{\rightarrow} \mu$. In particular, for all $n \in \mathbb{N}, E_{m} \subset\left\{y_{n}=\psi\right\}$ for $m$ large enough with $y_{n}=S\left(u_{n}\right)$, where the sequence $\left\{u_{n}\right\}$ is given by Lemma 5.4.

Now, we consider the sequence $\lambda_{m}:=\infty_{E_{m}}+\infty_{A_{s}(u)}$. By Theorem 3.16, we conclude that $\lambda_{m} \stackrel{\gamma}{\rightarrow} \lambda$ as $m \rightarrow \infty$. Fix $n \in \mathbb{N}$. Then Theorem 4.3 implies that $L_{\lambda_{m}} \in \partial_{B}^{s s} S\left(u_{n}\right)$ for 
all but finitely many $m \in \mathbb{N}$. Thus, the set inclusion $\partial_{B}^{s s} S(u) \subset \partial_{B}^{s w} S(u)$ and property (iii) from Proposition 2.11 imply that $L_{\lambda} \in \partial_{B}^{s w} S\left(u_{n}\right)$ for all $n \in \mathbb{N}$. Applying Proposition 2.11 once more, we obtain that $L_{\lambda} \in \partial_{B}^{s w} S(u)$ and the claim follows.

Now, we are able to give the main result of this section.

Theorem 5.6. Let $u \in H^{-1}(\Omega)$ be given such that the assumptions of Lemma 5.4 are satisfied. Then, (14) holds with equality, i.e.,

$$
\partial_{B}^{s w} S(u)=\left\{L_{\mu} \mid \mu \in \mathcal{M}_{0}(\Omega), \mu(I(u))=0 \text { and } \mu=+\infty \text { on } A_{s}(u)\right\} .
$$

Proof. Let $\mu \in \mathcal{M}_{0}(\Omega)$ with $\mu(I(u))=0$ and $\mu=\infty$ on $A_{s}(u)$. By Lemma 3.10 we find an increasing sequence $\left\{\mu_{m}\right\}$ of Radon measures with $\mu_{m} \stackrel{\gamma}{\rightarrow} \mu$. Since $\mu_{m} \leq \mu$, it holds $\mu_{m}(I(u))=0$. Thus, by Lemma 5.5 , the measure $\lambda_{m}:=\mu_{m}+\infty_{A_{s}(u)}$ satisfies

$$
L_{\lambda_{m}} \in \partial_{B}^{s w} S(u)
$$

Furthermore, Theorem 3.16 implies that $\lambda_{m} \stackrel{\gamma}{\rightarrow} \mu$ as $m \rightarrow \infty$. The closedness property of $\partial_{B}^{s w} S$, see Proposition 2.11, implies that $L_{\mu} \in \partial_{B}^{s w} S(u)$.

\section{The weak-weak generalized derivative}

By means of an example, we show that $\partial_{B}^{w w} S(u)$ can be surprisingly large. In fact, we have seen that for a Gâteaux point $u \in D_{S}$ we have $\partial_{B}^{s s} S(u)=\partial_{B}^{w s} S(u)=\partial_{B}^{s w} S(u)=\left\{S^{\prime}(u)\right\}$, see Theorems 4.3 and 4.4 and Lemma 5.1. However, we will see that $\partial_{B}^{w w} S(u)$ might not be a singleton for $u \in D_{S}$.

We use the classical construction of [Cioranescu, Murat, 1997]. Therein, the authors construct a sequence $\Omega_{n}$ of open subsets of $\Omega$ such that the solution operators $L_{\Omega_{n}}$ of

$$
-\Delta y_{n}=f \quad \text { in } \Omega_{n}
$$

converge in WOT to the solution operator $L_{c}$ of

$$
-\Delta y+c y=f \quad \text { in } \Omega
$$

for a positive constant $c>0$. We define $y=L_{c} 1$ and $y_{n}=L_{\Omega_{n}} 1$. This yields $y_{n} \rightarrow y$. We fix the obstacle $\psi:=0$ and set $u_{n}:=-\Delta y_{n}-2^{-n} \chi_{\Omega \backslash \Omega_{n}}, u:=-\Delta y$. Then, it is clear that $y=S(u), y_{n}=S\left(u_{n}\right)$ and $u_{n} \rightarrow u$. Since $A(u)=_{q} \emptyset$, we have $u \in D_{S}$. Similarly, we have $A\left(u_{n}\right)=_{q}\left\{y_{n}=0\right\}=_{q} \Omega \backslash \Omega_{n}$. From $\xi_{n}:=-\Delta y_{n}-u_{n}=2^{-n} \chi_{\Omega \backslash \Omega_{n}}$, we have $A_{s}\left(u_{n}\right)={ }_{q} \mathrm{f}-\operatorname{supp}\left(\xi_{n}\right)={ }_{q} \Omega \backslash \Omega_{n}$, since $\Omega \backslash \Omega_{n}$ is a finite union of balls (by construction). Thus, $u_{n} \in D_{S}$ and $S^{\prime}\left(u_{n}\right)=L_{\Omega_{n}}$. By construction, $L_{\Omega_{n}} \stackrel{\text { WOT }}{\longrightarrow} L_{c}$. Hence, $L_{c} \in \partial_{B}^{w w} S(u)$ although $u \in D_{S}$. 


\section{Stationarity systems for the optimal control of the obstacle problem}

In this section, we consider the optimal control of the obstacle problem with control constraints

$$
\text { Minimize } J(y, u) \text { with } y=S(u) \text { and } u \in U_{\text {ad }} \text {. }
$$

Here, $J: H_{0}^{1}(\Omega) \times L^{2}(\Omega) \rightarrow \mathbb{R}$ is given. We assume that $J$ is Fréchet differentiable with partial derivatives $J_{y}$ and $J_{u}$. The admissible set $U_{\text {ad }} \subset L^{2}(\Omega)$ is assumed to be closed and convex. We denote by $(y, u) \in H_{0}^{1}(\Omega) \times U_{\text {ad }}$ a local minimizer of (17). A formal calculation leads to the stationarity systems

$$
0 \in L^{\star} J_{y}(y, u)+J_{u}(y, u)+\mathcal{N}_{U_{\mathrm{ad}}}(u) \quad \text { for some } L \in \partial_{B}^{s s} S(u)
$$

and

$$
0 \in L^{\star} J_{y}(y, u)+J_{u}(y, u)+\mathcal{N}_{U_{\mathrm{ad}}}(u) \quad \text { for some } L \in \partial_{B}^{s w} S(u),
$$

where $\mathcal{N}_{U_{\text {ad }}}(u)$ denotes the normal cone (in the sense of convex analysis) of $U_{\text {ad }}$ at $u$. The goal of this section is the interpretation of these systems and a comparison with known optimality systems for (17). For the discussion of (19), we will assume that the characterization (16) holds. Recall that this is the case if $y$ and $\psi$ feature some additional regularity, see Theorem 5.6.

At this point, it is not clear whether any of these stationarity conditions is necessary for local optimality. If we would have defined the solution operator $S$ from $L^{2}(\Omega)$ to $H_{0}^{1}(\Omega)$, then (19) would imply that 0 belongs to the sum of the Bouligand subdifferential of the reduced objective $j(u):=J(S(u), u)$ at the point $u$ and the normal cone of $U_{\text {ad }}$ at $u$, see the discussion in [Christof et al., 2018, Section 4.2]. However, the derivation of the generalized derivatives for $S: L^{2}(\Omega) \rightarrow H_{0}^{1}(\Omega)$ is much more difficult and postponed to future work.

We start by the interpretation of (18).

Lemma 7.1. The condition (18) is equivalent to the existence of a quasi-closed set $A$ with $A_{s}(u) \subset_{q} A \subset_{q} A(u)$ and of $p \in H_{0}^{1}(\Omega), \nu \in H^{-1}(\Omega), \lambda \in \mathcal{N}_{U_{\text {ad }}}(u)$ such that

$$
\begin{aligned}
p+J_{u}(y, u)+\lambda & =0, & & p \in H_{0}^{1}(\Omega \backslash A), \\
-\Delta p+\nu & =J_{y}(y, u), & \nu & \in H^{-1}(\Omega) \text { with }\langle\nu, v\rangle=0 \text { for all } v \in H_{0}^{1}(\Omega \backslash A)
\end{aligned}
$$

hold.

Proof. Let (18) be satisfied with some $L \in \partial_{B}^{s s} S(u)$. By Theorem 4.3, there exists a quasi-closed set $A$ with $A_{s}(u) \subset_{q} A \subset_{q} A(u)$ and $L=L_{\Omega \backslash A}$. Then, it is clear that $p:=L^{\star} J_{y}(y, u)=L J_{y}(y, u), \nu:=J_{y}(y, u)+\Delta p$ and $\lambda:=-p-J_{u}(y, u)$ satisfy the above system.

The converse direction follows similarly. 
We note that the condition of Lemma 7.1 is a rather restrictive version of the system of M-stationarity in [G. Wachsmuth, 2016, Section 1.4], just use $\hat{A}_{s}:={ }_{q} A \backslash A_{s}(u), \hat{B}:={ }_{q} \emptyset$ and $\hat{A}:={ }_{q} A(u) \backslash A$ therein.

The interpretation of (19) is much more challenging and interesting.

Lemma 7.2. The condition (19) implies the existence of $p \in H_{0}^{1}(\Omega), \nu \in H^{-1}(\Omega)$, $\lambda \in \mathcal{N}_{U_{\text {ad }}}(u)$ such that

$$
\begin{aligned}
p+J_{u}(y, u)+\lambda & =0 \quad p \in H_{0}^{1}\left(\Omega \backslash A_{s}(u)\right) \\
-\Delta p+\nu & =J_{y}(y, u) \quad \nu \in H^{-1}(\Omega) \text { with }\langle\nu, v\rangle=0 \forall v \in H_{0}^{1}(\Omega \backslash A(u)) \\
\langle\nu, p \varphi\rangle & \geq 0 \quad \forall \varphi \in W^{1, \infty}(\Omega)^{+} .
\end{aligned}
$$

Conversely, if this system holds, if (16) holds and if there exists $\mu \in \mathcal{M}_{0}(\Omega)$ such that $\nu=p \mu$ in the sense $p \in L_{\mu}^{2}(\Omega)$ and

$$
\langle\nu, w\rangle=\int_{\Omega} p w \mathrm{~d} \mu \quad \forall w \in H_{0}^{1}(\Omega) \cap L_{\mu}^{2}(\Omega),
$$

then (19) is satisfied.

Proof. " $(19) \Rightarrow(20)$ ": Let (19) be satisfied by some $L \in \partial_{B}^{s w} S(u)$. From (14), there is $\mu \in \mathcal{M}_{0}(\Omega)$ with $L=L_{\mu}, \mu(I(u))=0$ and $\mu=+\infty$ on $A_{s}(u)$. We define $p:=$ $L^{\star} J_{y}(y, u)=L J_{y}(y, u), \nu:=J_{y}(y, u)+\Delta p$ and $\lambda:=-p-J_{u}(y, u)$. Then, $p=0$ q.e. on $A_{s}(u)$, i.e., $p \in H_{0}^{1}\left(\Omega \backslash A_{s}(u)\right)$.

By definition of $\nu$ and $p$, we have

$$
\nu=J_{y}(y, u)+\Delta p=p \mu .
$$

For $v \in H_{0}^{1}(\Omega \backslash A(u))=H_{0}^{1}(I(u))$, we have $v \in L_{\mu}^{2}(\Omega)$ and we get

$$
\langle\nu, v\rangle=\int_{\Omega} p v \mathrm{~d} \mu=0
$$

since $\mu=0$ on $I(u)$ and $v$ lives only on $I(u)$.

It remains to show $\langle\nu, p \varphi\rangle=0$ for all $\varphi \in W^{1, \infty}(\Omega)^{+}$. We have $p \varphi \in H_{0}^{1}(\Omega)$ and the pointwise boundedness of $\varphi$ gives $p \varphi \in L_{\mu}^{2}(\Omega)$. Thus,

$$
\langle\nu, p \varphi\rangle=\int_{\Omega} p^{2} \varphi \mathrm{d} \mu \geq 0 .
$$

This shows that the above system is satisfied by $p$ and $\nu$.

" $(20) \Rightarrow(19) "$ : To prove the converse direction, let $p, \nu, \lambda$ and $\mu$ be given as in the assertion of the lemma. We will modify $\mu$ to construct another measure $\mu_{2} \in \mathcal{M}_{0}(\Omega)$, which satisfies the conditions on the right-hand side of (16), that is, $\mu_{2}(I(u))=0$ and $\mu_{2}=+\infty$ on $A_{s}(u)$. First, we will set the measure to $+\infty$ in $A_{s}(u)$. Since $\{p=$ $0\} \backslash I(u) \supset_{q} A_{s}(u)$, we define $\mu_{1}:=\mu+\infty_{\{p=0\} \backslash I(u)}$. We check that $\nu=p \mu_{1}$. Obviously, 
$p \in L_{\mu_{1}}^{2}(\Omega)$ since $p=0$ q.e. on $\{p=0\} \backslash I(u)$. Furthermore, for $w \in H_{0}^{1}(\Omega) \cap L_{\mu_{1}}^{2}(\Omega)$, we have $w \in L_{\mu}^{2}(\Omega)$, thus

$$
\langle\nu, w\rangle=\int_{\Omega} p w \mathrm{~d} \mu=\int_{\Omega} p w \mathrm{~d} \mu_{1}
$$

since $\int_{\Omega} p w \mathrm{~d} \infty_{\{p=0\} \backslash I(u)}=0$.

Next, we define the Borel measure $\mu_{2}(B):=\mu_{1}(B \backslash I(u))$. Then, $\mu_{2}(I(u))=0$. It remains to show that we still have $\nu=p \mu_{2}$. The condition $p \in L_{\mu_{2}}^{2}(\Omega)$ is clear.

We use Lemmas 2.4 and 3.13 to obtain

$$
\begin{aligned}
{\overline{H_{0}^{1}(\Omega) \cap L_{\mu_{1}}^{2}(\Omega)+H_{0}^{1}(I(u))}}^{H_{0}^{1}(\Omega)} & =\overline{{\overline{H_{0}^{1}(\Omega) \cap L_{\mu_{1}}^{2}(\Omega)}}^{H_{0}^{1}(\Omega)}+H_{0}^{1}(I(u))}{ }^{1}(\Omega) \\
& =\overline{H_{0}^{1}\left(\left\{w_{\mu_{1}}>0\right\}\right)+H_{0}^{1}(I(u))} H_{0}^{1}(\Omega) \\
& =H_{0}^{1}\left(\left\{w_{\mu_{1}}>0\right\} \cup I(u)\right) .
\end{aligned}
$$

For all Borel sets $B \subset \Omega$ with $\operatorname{cap}(B \cap\{p=0\} \backslash I(u))>0$, we have

$$
\mu_{2}(B)=\mu_{1}(B \backslash I(u))=\mu(B \backslash I(u))+\infty_{\{p=0\} \backslash I(u)}(B \backslash I(u))=+\infty .
$$

Thus, $w_{\mu_{2}}=0$ q.e. on $\{p=0\} \backslash I(u)$. By taking complements, this leads to

$$
\left\{w_{\mu_{2}}>0\right\} \subset_{q} \Omega \backslash(\{p=0\} \backslash I(u))=_{q} \Omega \backslash(\{p=0\} \cap A(u))=_{q}\{p \neq 0\} \cup I(u) .
$$

Moreover, from $p \in L_{\mu_{1}}^{2}(\Omega)$ and Lemma 3.12, we obtain

$$
\left\{w_{\mu_{2}}>0\right\} \subset_{q}\left\{w_{\mu_{1}}>0\right\} \cup I(u) .
$$

By combining (22), (23) and Lemma 3.12, we find that every $v \in H_{0}^{1}(\Omega)^{+} \cap L_{\mu_{2}}^{2}(\Omega)$ belongs to $H_{0}^{1}\left(\left\{w_{\mu_{1}}>0\right\} \cup I(u)\right)^{+}$and, therefore, there exist sequences $\left\{v_{n}^{(1)}\right\}_{n \in \mathbb{N}} \subset$ $H_{0}^{1}(\Omega) \cap L_{\mu_{1}}^{2}(\Omega)$ and $\left\{v_{n}^{(2)}\right\}_{n \in \mathbb{N}} \subset H_{0}^{1}(I(u))$ with $v_{n}^{(1)}+v_{n}^{(2)} \rightarrow v$ in $H_{0}^{1}(\Omega)$. Further, from the second assertions of Lemmas 2.4 and 3.13, it can be seen that these sequences can be chosen such that additionally $0 \leq v_{n}^{(1)}+v_{n}^{(2)} \leq v$ q.e. on $\Omega$ for all $n \in \mathbb{N}$. We can extract a subsequence (without relabeling), such that $v_{n}^{(1)}+v_{n}^{(2)} \rightarrow v$ pointwise q.e., thus, pointwise $\mu_{2}$-a.e. Since $v \in L_{\mu_{2}}^{2}(\Omega)$, the dominated convergence theorem implies $v_{n}^{(1)}+v_{n}^{(2)} \rightarrow v$ in $L_{\mu_{2}}^{2}(\Omega)$.

By construction, the functional $K: H_{0}^{1}(\Omega) \cap L_{\mu_{2}}^{2}(\Omega) \rightarrow \mathbb{R}$, given by

$$
K(w):=\langle\nu, w\rangle-\int_{\Omega} p w \mathrm{~d} \mu_{2}
$$

vanishes on $H_{0}^{1}(I(u))$.

Next, we show that $K$ vanishes also on $H_{0}^{1}(\Omega) \cap L_{\mu_{1}}^{2}(\Omega)$. We take $w \in H_{0}^{1}(I(u))$ with $0 \leq w \leq 1$ and $\{w>0\}={ }_{q} I(u)$. Then, $\tilde{w}=\max (\min (w, p),-w)$ satisfies $\tilde{w} \in H_{0}^{1}(I(u)) \cap L_{\mu_{1}}^{2}(\Omega)$, since $|\tilde{w}| \leq|p| \in L_{\mu_{1}}^{2}(\Omega)$. Hence, (20b) and (21) imply

$$
0=\langle\nu, \tilde{w}\rangle=\int_{\Omega} p \tilde{w} \mathrm{~d} \mu_{1} .
$$


Now, $p \tilde{w} \geq 0$ and $\{p \tilde{w}>0\}={ }_{q}\{|p| \neq 0\} \cap I(u)$. This shows $\mu_{1}(I(u) \cap\{p \neq 0\})=0$. Thus, for arbitrary $w \in H_{0}^{1}(\Omega) \cap L_{\mu_{1}}^{2}(\Omega)$, we have

$$
\langle\nu, w\rangle=\int_{\Omega} p w \mathrm{~d} \mu_{1}=\int_{\Omega \backslash I(u)} p w \mathrm{~d} \mu_{1}=\int_{\Omega \backslash I(u)} p w \mathrm{~d} \mu_{2}=\int_{\Omega} p w \mathrm{~d} \mu_{2} .
$$

Hence, $K$ vanishes on $H_{0}^{1}(\Omega) \cap L_{\mu_{1}}^{2}(\Omega)$.

Further, $K$ is linear and continuous w.r.t. the space $H_{0}^{1}(\Omega) \cap L_{\mu_{2}}^{2}(\Omega)$. Thus,

$$
K(v)=\lim _{n \rightarrow \infty} K\left(v_{n}^{(1)}+v_{n}^{(2)}\right)=0 .
$$

Hence, $K$ vanishes on $H_{0}^{1}(\Omega)^{+} \cap L_{\mu_{2}}^{2}(\Omega)$ and, by linearity, on the entire space $H_{0}^{1}(\Omega) \cap$ $L_{\mu_{2}}^{2}(\Omega)$. This shows $\nu=p \mu_{2}$.

Now, for $v \in H_{0}^{1}(\Omega) \cap L_{\mu_{2}}^{2}(\Omega)$, we have

$$
\int_{\Omega} p v \mathrm{~d} \mu_{2}=\langle\nu, v\rangle=\left\langle J_{y}(y, u), v\right\rangle-\int_{\Omega} \nabla p \nabla v \mathrm{~d} x .
$$

This shows $p=L_{\mu_{2}} J_{y}(y, u)=L_{\mu_{2}}^{\star} J_{y}(y, u)$. Hence, (19) is satisfied.

Some remarks concerning Lemma 7.2 are in order. Under some regularity assumptions on the data and on the objective of the control problem (17), it was shown in [Schiela, D. Wachsmuth, 2013] that the system (20) is satisfied at every local minimizer, see also the comparison in [G. Wachsmuth, 2016, Lemma 4.6].

Surprisingly, the technique of [Schiela, D. Wachsmuth, 2013] even provides the additional condition $\nu=p \mu$ after closer inspection. Indeed, (by using the notation of [Schiela, D. Wachsmuth, 2013]), the adjoint state $p_{c}$ associated to a regularized problem solves the semilinear equation

$$
-\Delta p_{c}+c \max _{c}^{\prime}\left(\bar{\lambda}+\left(y_{c}-\psi\right)\right) p_{c}=J_{y}\left(y_{c}, u_{c}\right) .
$$

Here, $c>0$ is a penalty parameter which will go to $\infty$. Since the function $\max _{c}$ is monotonically increasing, we have $c \max _{c}^{\prime}\left(\bar{\lambda}+\left(y_{c}-\psi\right)\right) \in \mathcal{M}_{0}(\Omega)$. Theorem 3.8 implies that (along a subsequence) $c \max _{c}^{\prime}\left(\bar{\lambda}+\left(y_{c}-\psi\right)\right) \stackrel{\gamma}{\rightarrow} \mu$ for some $\mu \in \mathcal{M}_{0}(\Omega)$ as $c \rightarrow \infty$. Thus, the weak convergence $p_{c} \rightarrow p$ in $H_{0}^{1}(\Omega)$, together with $y_{c} \rightarrow y$ in $H_{0}^{1}(\Omega)$ and $u_{c} \rightarrow u$ in $L^{2}(\Omega)$, yields that the limit $p$ satisfies

$$
-\Delta p+\mu p=J_{y}(y, u) .
$$

Hence, $\nu=p \mu$ in the sense of Lemma 7.2. This reasoning and the results of [Schiela, D. Wachsmuth, 2013] imply that (19) is indeed satisfied by every local minimizer of (17), whenever (16) holds. 


\section{Conclusion}

In this work we have shown that the generalized derivatives of the solution operator $S$ of the obstacle problem are solution operators of relaxed Dirichlet problems. In the case that the strong operator topology is considered, the limit is a solution operator associated to a quasi-open subset of $\Omega$, whereas the usage of the weak operator topology needs the notion of solution operators associated with capacitary measures. By considering optimality systems corresponding to the generalized derivatives of $S$, we have seen that the notion of C-stationarity from [Schiela, D. Wachsmuth, 2013] can be strengthened to a system including a capacitary measure.

\section{Acknowledgments}

The authors would like to thank Giuseppe Buttazzo for pointing out the result of [Bucur et al., 1999, Lemma 4.12] which led to the discovery of Theorem 3.16. Further, Constantin Christof pointed out the result of Theorem $3.9\left(\mathrm{f}-\operatorname{supp}(1+\Delta w)={ }_{q} \Omega \backslash O\right.$ for $\left.w=L_{O}(1)\right)$ and this is gratefully acknowledged. He gave a different, interesting proof based on differentiability properties of the obstacle problem and this proof might appear elsewhere.

This work is supported by DFG grants UL158/10-1 and WA3636/4-1 within the Priority Program SPP 1962 (Non-smooth and Complementarity-based Distributed Parameter Systems: Simulation and Hierarchical Optimization).

\section{References}

Attouch, Hedy; Buttazzo, Giuseppe; Michaille, Gérard (2014). Variational analysis in Sobolev and BV spaces. Second. Vol. 17. MOS-SIAM Series on Optimization. Applications to PDEs and optimization. Society for Industrial and Applied Mathematics (SIAM), Philadelphia, PA; Mathematical Optimization Society, Philadelphia, PA, pp. xii+793. ISBN: 978-1-611973-47-1. DOI: 10.1137/1.9781611973488.

Bonnans, Joseph Frédéric; Shapiro, Alexander (2000). Perturbation analysis of optimization problems. New York, NY: Springer, pp. xviii + 601. ISBN: 0-387-98705-3/hbk.

Bucur, Dorin; Buttazzo, Giuseppe (2005). Variational methods in shape optimization problems. Basel: Birkhäuser, pp. viii +216 . ISBN: 0-8176-4359-1/hbk.

Bucur, Dorin; Buttazzo, Giuseppe; Trebeschi, Paola (1999). "An existence result for optimal obstacles". In: Journal of Functional Analysis 162.1, pp. 96-119. ISSN: 00221236. DOI: 10.1006/jfan.1998.3363.

Buttazzo, Giuseppe; Dal Maso, Gianni (1991). "Shape optimization for Dirichlet problems: Relaxed formulation and optimality conditions". In: Applied Mathematics and Optimization 23.1, pp. 17-49. ISSN: 0095-4616; 1432-0606/e. DOI: 10.1007/BF01442391.

Chipot, Michel; Dal Maso, Gianni (1992). "Relaxed shape optimization: the case of nonnegative data for the Dirichlet problem". In: Advances in Mathematical Sciences and Applications 1.1, pp. 47-81. ISSN: 1343-4373. 
Christof, Constantin; Clason, Christian; Meyer, Christian; Walther, Stephan (2018). "Optimal Control of a Non-smooth Semilinear Elliptic Equation". In: Mathematical Control \& Related Fields 8.1, pp. 247-276. DOI: 10.3934/mcrf. 2018011.

Cioranescu, Doina; Murat, François (1997). "A strange term coming from nowhere". In: Topics in the mathematical modelling of composite materials. Vol. 31. Progr. Nonlinear Differential Equations Appl. Boston, MA: Birkhäuser Boston, pp. 45-93.

Dal Maso, Gianni (1983). "On the integral representation of certain local functionals." In: Ricerche di Matematica 32, pp. 85-113. ISSN: 0035-5038; 1827-3491/e.

Dal Maso, Gianni (1987). "Г-convergence and $\mu$-capacities". In: Annali della Scuola Normale Superiore di Pisa. Classe di Scienze. Serie IV 14.3, 423-464 (1988). ISSN: 0391-173X. URL: http://www.numdam.org/item?id=ASNSP_1987_4_14_3_423_0.

Dal Maso, Gianni; Garroni, Adriana (1994). "New results on the asymptotic behavior of Dirichlet problems in perforated domains". In: Mathematical Models and Methods in Applied Sciences 4.3, pp. 373-407. ISSN: 0218-2025. DOI: 10.1142/S0218202594000224.

Dal Maso, Gianni; Garroni, Adriana (1997). "The capacity method for asymptotic Dirichlet problems". In: Asymptotic Analysis 15.3-4, pp. 299-324. ISSN: 0921-7134.

Dal Maso, Gianni; Malusa, Annalisa (1995). "Approximation of relaxed Dirichlet problems by boundary value problems in perforated domains". In: Proceedings of the Royal Society of Edinburgh. Section A. Mathematics 125.1, pp. 99-114. ISSN: 0308-2105. DOI: 10.1017/S0308210500030778.

Dal Maso, Gianni; Mosco, Umberto (1986). "Wiener criteria and energy decay for relaxed Dirichlet problems". In: Archive for Rational Mechanics and Analysis 95, pp. 345-387. ISSN: 0003-9527; 1432-0673/e. DOI: $10.1007 / \mathrm{BF} 00276841$.

Dal Maso, Gianni; Mosco, Umberto (1987). "Wiener's criterion and $\Gamma$-convergence". In: Applied Mathematics and Optimization 15.1, pp. 15-63. ISSN: 0095-4616. DOI: 10.1007/BF01442645.

Dal Maso, Gianni; Murat, François (2004). "Asymptotic behaviour and correctors for linear Dirichlet problems with simultaneously varying operators and domains". In: Annales de l'Institut Henri Poincaré. Analyse Non Linéaire 21.4, pp. 445-486. ISSN: 0294-1449. DOI: 10.1016/j . anihpc.2003.05.001.

Delfour, Michel C.; Zolésio, Jean-Paul (2011). Shapes and geometries. Metrics, analysis, differential calculus, and optimization. Second. Philadelphia, PA: Society for Industrial and Applied Mathematics (SIAM), pp. xxiii + 622. ISBN: 978-0-898719-36-9/hbk; 978-0-89871-982-6/ebook. DOI: 10.1137/1.9780898719826.

Harder, Felix; Wachsmuth, Gerd (2018). "Comparison of Optimality Systems for the Optimal Control of the Obstacle Problem". In: GAMM-Mitteilungen 40.4, pp. 312-338. DOI: $10.1002 /$ gamm . 201740004.

Heinonen, Juha; Kilpeläinen, Tero; Martio, Olli (1993). Nonlinear potential theory of degenerate elliptic equations. Oxford Mathematical Monographs. Oxford Science Publications. New York: The Clarendon Press Oxford University Press. ISBN: 0-19-853669-0.

Kilpeläinen, Tero; Malý, Jan (1992). "Supersolutions to degenerate elliptic equation on quasi open sets". In: Communications in Partial Differential Equations 17.3-4, pp. 371-405. ISSN: 0360-5302. DOI: 10.1080/03605309208820847. 
Klatte, Diethard; Kummer, Bernd (2002). Nonsmooth equations in optimization. Regularity, calculus, methods and applications. Dordrecht: Kluwer Academic Publishers, pp. xxviii + 329. ISBN: 1-4020-0550-4/hbk.

Mignot, Fulbert (1976). "Contrôle dans les inéquations variationelles elliptiques". In: Journal of Functional Analysis 22, pp. 130-185. ISSN: 0022-1236. DOI: 10.1016/00221236 (76) 90017-3.

Outrata, Jiři; Kočvara, Michal; Zowe, Jochem (1998). Nonsmooth approach to optimization problems with equilibrium constraints. Theory, applications and numerical results. Dordrecht: Kluwer Academic Publishers, pp. xxi + 273. ISBN: 0-7923-5170-3.

Rauls, Anne-Therese; Ulbrich, Stefan (2018). Subgradient computation for the solution operator of the obstacle problem. Tech. rep. Preprint SPP1962-056. URL: https:// spp1962.wias-berlin.de/preprints/056.pdf.

Rudin, Walter (1987). Real and Complex Analysis. Mathematics series. McGraw-Hill. ISBN: 9780071002769.

Schiela, Anton; Wachsmuth, Daniel (2013). "Convergence analysis of smoothing methods for optimal control of stationary variational inequalities with control constraints". In: ESAIM: Mathematical Modelling and Numerical Analysis 47.3, pp. 771-787. DOI: 10.1051/m2an/2012049.

Stollmann, Peter (1993). "Closed ideals in Dirichlet spaces". In: Potential Analysis. An International Journal Devoted to the Interactions between Potential Theory, Probability Theory, Geometry and Functional Analysis 2.3, pp. 263-268. ISSN: 0926-2601. DOI: 10.1007/BF01048510.

Velichkov, Bozhidar (2015). Existence and regularity results for some shape optimization problems. Vol. 19. Tesi. Scuola Normale Superiore di Pisa (Nuova Series) [Theses of Scuola Normale Superiore di Pisa (New Series)]. Edizioni della Normale, Pisa, pp. xvi+349. ISBN: 978-88-7642-526-4; 978-88-7642-527-1. DOI: 10.1007/978-88-7642$527-1$.

Wachsmuth, Gerd (2014). "Strong stationarity for optimal control of the obstacle problem with control constraints". In: SIAM Journal on Optimization 24.4, pp. 1914-1932. ISSN: 1052-6234; 1095-7189/e. DOI: $10.1137 / 130925827$.

Wachsmuth, Gerd (2016). "Towards M-stationarity for optimal control of the obstacle problem with control constraints". In: SIAM Journal on Control and Optimization 54.2, pp. 964-986. DOI: $10.1137 / 140980582$. 